MPP-2005-24

hep-ph/0506005

\title{
Four-fermion production at $\gamma \gamma$ colliders: 2. Radiative corrections in double-pole approximation
}

\author{
A. Bredenstein, S. Dittmaier and M. Roth \\ Max-Planck-Institut für Physik (Werner-Heisenberg-Institut) \\ D-80805 München, Germany
}

\begin{abstract}
:
The $\mathcal{O}(\alpha)$ electroweak radiative corrections to $\gamma \gamma \rightarrow \mathrm{WW} \rightarrow 4 f$ within the electroweak Standard Model are calculated in double-pole approximation (DPA). Virtual corrections are treated in DPA, leading to a classification into factorizable and non-factorizable contributions, and real-photonic corrections are based on complete lowest-order matrix elements for $\gamma \gamma \rightarrow 4 f+\gamma$. Soft and collinear singularities appearing in the virtual and real corrections are combined alternatively in two different ways, namely by using the dipole subtraction method or by applying phase-space slicing. The radiative corrections are implemented in a Monte Carlo generator called CofFER $\gamma \gamma^{\dagger}$, which optionally includes anomalous triple and quartic gauge-boson couplings in addition and performs a convolution over realistic spectra of the photon beams. A detailed survey of numerical results comprises $\mathcal{O}(\alpha)$ corrections to integrated cross sections as well as to angular, energy, and invariant-mass distributions. Particular attention is paid to the issue of collinear-safety in the observables.
\end{abstract}

June 2005

\footnotetext{
${ }^{\dagger}$ The computer code can be obtained from the authors upon request.
} 


\section{Introduction}

As an option at a future $\mathrm{e}^{+} \mathrm{e}^{-}$linear collider, a photon (or $\left.\gamma \gamma\right)$ collider [1] found considerable interest in recent years. It could provide us with information about new physics phenomena, such as properties of Higgs bosons or of new particles, which is in many respects complementary in the $\mathrm{e}^{+} \mathrm{e}^{-}$and $\gamma \gamma$ modes (see, e.g., Refs. [1,2] and references therein). Moreover, a $\gamma \gamma$ collider is a true $\mathrm{W}$-boson-pair factory, owing to the extremely high W-pair cross section, which tends to a constant of about $80 \mathrm{pb}$ in the high-energy limit (in the absence of phase-space cuts), opening the possibility of precision studies in the sector of electroweak gauge bosons. Either way, whether one is interested in W-boson precision physics or in the search for new phenomena, precise predictions for W-pair production are indispensable for signal and background studies.

In our previous work [3] we have made the first step towards a precision calculation for the processes $\gamma \gamma \rightarrow \mathrm{WW} \rightarrow 4 f(+\gamma)$ by constructing a Monte Carlo event generator for lowest-order predictions based on complete matrix elements for the processes $\gamma \gamma \rightarrow 4 f$ and $\gamma \gamma \rightarrow 4 f \gamma$. The possibility to convolute the cross sections with realistic photon beam spectra is offered upon using the parametrization of CoMPAZ [4]. The Standard Model (SM) predictions were successfully compared to results obtained with the multi-purpose packages Whizard [5] and MAdGraPH [6]. Moreover, we included an effective $\gamma \gamma \mathrm{H}$ coupling, which is induced by loop diagrams, as well as anomalous triple and quartic gauge-boson couplings. The former is needed for studying Higgs production in the $s$ channel. An analysis of anomalous gauge-boson couplings in $\gamma \gamma \rightarrow \mathrm{WW}$ provides direct information on the $\gamma \mathrm{WW}$ and $\gamma \gamma \mathrm{WW}$ interactions without interference from the Z-boson sector. Both the Higgs resonance in the $s$-channel and the more direct access to the $\gamma \mathrm{WW}$ and $\gamma \gamma \mathrm{WW}$ interactions are complementary to the situation in $\mathrm{e}^{+} \mathrm{e}^{-}$annihilation.

In this paper we extend our lowest-order calculation [3] for $\gamma \gamma \rightarrow 4 f$ by including the electroweak radiative corrections of $\mathcal{O}(\alpha)$ to the W-pair channels $\gamma \gamma \rightarrow \mathrm{WW} \rightarrow 4 f$ in the so-called "double-pole approximation" (DPA). The DPA extracts those contributions of the $\mathcal{O}(\alpha)$ corrections that are enhanced by two resonant W-boson propagators, i.e. it represents the leading term in an expansion of the cross section about the two $\mathrm{W}$-propagator poles. Note that tree-level diagrams for $\gamma \gamma \rightarrow 4 f$ with at most one resonant $\mathrm{W}$ boson are suppressed w.r.t. the doubly-resonant $\gamma \gamma \rightarrow \mathrm{WW}$ signal by a factor of $\mathcal{O}\left(\Gamma_{\mathrm{W}} / M_{\mathrm{W}}\right) \sim \mathcal{O}(\alpha)$. Consequently, predictions based on full lowest-order matrix elements for $\gamma \gamma \rightarrow 4 f$ and $\mathcal{O}(\alpha)$ corrections for $\gamma \gamma \rightarrow \mathrm{WW} \rightarrow 4 f$ in DPA should be precise up to terms of $\mathcal{O}\left(\alpha / \pi \times \Gamma_{\mathrm{W}} / M_{\mathrm{W}}\right)$, since corrections typically involve the factor $\alpha / \pi$. Including a quite conservative numerical safety factor, the relative uncertainty should thus

be $\lesssim 0.5 \%$ for such predictions, as long as neglected effects are not additionally enhanced. The naive error estimate can, in particular, be spoiled by the occurrence of large scale ratios, which exist, e.g., near production thresholds or at very high energies. The estimate has recently been confirmed for $\mathrm{e}^{+} \mathrm{e}^{-} \rightarrow \mathrm{WW} \rightarrow 4 f$ with centre-of-mass (CM) energies $170 \mathrm{GeV} \lesssim \sqrt{s} \lesssim 300 \mathrm{GeV}$ by comparing a full $\mathcal{O}(\alpha)$ calculation [7,8] with the corresponding DPA predictions provided by RACOONWW $[9,10]$.

In detail, we apply the DPA only to the virtual corrections to $\gamma \gamma \rightarrow \mathrm{WW} \rightarrow 4 f$, while we base the real-photonic corrections on complete lowest-order matrix elements for $\gamma \gamma \rightarrow 4 f \gamma$. Apart from the treatment of IR (soft and collinear) singularities, we can use 
the calculation of the bremsstrahlung processes $\gamma \gamma \rightarrow 4 f \gamma$ for massless fermions described in Ref. [3]. The concept of the DPA was already described in Ref. [11] for the corrections to $\mathrm{e}^{+} \mathrm{e}^{-} \rightarrow \mathrm{WW} \rightarrow 4 f$ and later successfully applied to these processes in different versions $[9,10,12-14]$. We follow the strategy of RACOONWW $[9,10]$ and adapt it to $\gamma \gamma$ collisions where necessary. The virtual corrections in DPA can be naturally split into factorizable and non-factorizable contributions. The former comprise the corrections to on-shell Wpair production $[15-17]^{1}$ and the decay [19] of on-shell $\mathrm{W}$ bosons. The latter account for soft-photon exchange between the production and decay subprocesses; the known results for the non-factorizable corrections $[20,21]$ for $\mathrm{e}^{+} \mathrm{e}^{-} \rightarrow \mathrm{WW} \rightarrow 4 f$ can be taken over to $\gamma \gamma$ collisions with minor modifications. Although the basic building blocks for the virtual corrections exist in the literature, the combination into a complete set of $\mathcal{O}(\alpha)$ corrections in DPA has not been done yet for $\gamma \gamma \rightarrow \mathrm{WW} \rightarrow 4 f$.

The combination of virtual and real-photonic corrections is non-trivial for two reasons. First, the finite-fermion-mass effects have to be restored in the phase-space regions of collinear photon radiation off charged fermions, and the IR regularization for softphoton emission has to be implemented. To this end, we employ the dipole subtraction formalism for photon radiation $[22,23]$ as well as the more conventional phase-space slicing approach. The second subtlety concerns the fact that we apply the DPA only to the virtual corrections, but not to the real-photonic parts. Therefore, the cancellation of soft and collinear singularities has to be done carefully, in order to avoid mismatch.

The paper is organized as follows: After a brief outline of our strategy in the next section, in Section 3 we describe the actual calculation of the virtual corrections in DPA. Apart from the general concept, we give some details on an efficient way for a numerically stable evaluation, on renormalization issues, on the treatment of the $s$-channel Higgs resonance, and on an improved Born approximation used in the threshold region of W-pair production. Section 4 deals with the combination of virtual and real-photonic corrections; all relevant details for the application of dipole subtraction and phase-space slicing to the considered processes can be found there. Moreover, the differences in the evaluation of collinear-safe and non-collinear-safe observables are described. Our discussion of numerical results is presented in Section 5; besides integrated cross sections, we also discuss angular, energy, and invariant-mass distributions. A summary is given in Section 6, and the appendices provide further details on the evaluation of coefficient functions for the factorizable virtual corrections as well as on the generalization of the dipole subtraction method for non-collinear-safe observables.

\section{Strategy of the calculation}

We consider the process

$$
\begin{aligned}
\gamma\left(k_{1}, \lambda_{1}\right)+\gamma\left(k_{2}, \lambda_{2}\right) & \rightarrow \mathrm{W}^{+}\left(k_{+}, \lambda_{+}\right)+\mathrm{W}^{-}\left(k_{-}, \lambda_{-}\right) \\
& \rightarrow f_{1}\left(p_{1}, \sigma_{1}\right)+\bar{f}_{2}\left(p_{2}, \sigma_{2}\right)+f_{3}\left(p_{3}, \sigma_{3}\right)+\bar{f}_{4}\left(p_{4}, \sigma_{4}\right),
\end{aligned}
$$

where $k_{i}$ and $p_{i}$ denote the momenta and $\lambda_{i}$ and $\sigma_{i}$ the helicities of the corresponding particles.

\footnotetext{
${ }^{1}$ Radiative corrections to on-shell W-pair production, $\gamma \gamma \rightarrow \mathrm{WW}$, were also considered in Ref. [18].
} 
The lowest-order cross section $\mathrm{d} \sigma_{\text {Born }}^{\gamma \gamma \rightarrow 4 f}$, based on the complete matrix elements $\mathcal{M}_{\text {Born }}^{\gamma \gamma \rightarrow 4 f}$ with massless fermions, has been discussed in detail in Ref. [3]. Suppressing the averaging over the photon polarizations and the spin and colour summation for the final state in the notation, it reads

$$
\int \mathrm{d} \sigma_{\text {Born }}^{\gamma \gamma \rightarrow 4 f}=\frac{1}{2 s} \int \mathrm{d} \Phi_{4 f}\left|\mathcal{M}_{\text {Born }}^{\gamma \gamma \rightarrow 4 f}\right|^{2},
$$

with

$$
s=\left(k_{1}+k_{2}\right)^{2}, \quad s_{i j}=\left(p_{i}+p_{j}\right)^{2}, \quad i, j=1,2,3,4 .
$$

The variables $s_{i j}$ are introduced for later use.

In the following we focus on the radiative corrections of $\mathcal{O}(\alpha)$ which consist of virtual corrections $\mathrm{d} \sigma_{\text {virt }}^{\gamma \gamma \rightarrow 4 f}$ to the process (2.1) and real-photonic corrections $\mathrm{d} \sigma^{\gamma \gamma \rightarrow 4 f \gamma}$, originating from the process

$$
\begin{aligned}
\gamma\left(k_{1}, \lambda_{1}\right)+\gamma\left(k_{2}, \lambda_{2}\right) & \rightarrow \mathrm{W}^{+}\left(k_{+}, \lambda_{+}\right)+\mathrm{W}^{-}\left(k_{-}, \lambda_{-}\right)(+\gamma) \\
& \rightarrow f_{1}\left(p_{1}, \sigma_{1}\right)+\bar{f}_{2}\left(p_{2}, \sigma_{2}\right)+f_{3}\left(p_{3}, \sigma_{3}\right)+\bar{f}_{4}\left(p_{4}, \sigma_{4}\right)+\gamma\left(k, \lambda_{\gamma}\right) .
\end{aligned}
$$

Combining the different contributions we obtain the $\mathcal{O}(\alpha)$-corrected prediction for the cross section,

$$
\int \mathrm{d} \sigma=\int \mathrm{d} \sigma_{\text {Born }}^{\gamma \gamma \rightarrow 4 f}+\int \mathrm{d} \sigma_{\text {virt }}^{\gamma \gamma \rightarrow 4 f}+\int \mathrm{d} \sigma^{\gamma \gamma \rightarrow 4 f \gamma} .
$$

The real-photonic corrections $\mathrm{d} \sigma^{\gamma \gamma \rightarrow 4 f \gamma}$ are based on the full lowest-order matrix elements $\mathcal{M}_{\text {Born }}^{\gamma \gamma \rightarrow 4 f \gamma}$ of the process $\gamma \gamma \rightarrow 4 f \gamma$, which were calculated in Ref. [3] for massless fermions. In the limit of vanishing photon momentum $k$ (soft limit) or when the photon becomes collinear to an external charged fermion (collinear limit), the cross section diverges. Considering the process $\gamma \gamma \rightarrow 4 f \gamma$ with a visible photon (which is neither soft nor collinear), these singularities are removed by imposing appropriate phase-space cuts which are justified by the finite experimental resolution. For predictions of the $\gamma \gamma \rightarrow 4 f(\gamma)$ processes, i.e. with or without photon radiation, the singular phase-space regions of soft or collinear emission have to be integrated over. In this case the real corrections are combined with the virtual corrections which contain exactly the same singularities with opposite sign. The regularization of the singularities in the real corrections by small photon and fermion masses, $\lambda$ and $m_{f}$, as well as the matching with the singularities in the virtual corrections, is described in detail in Section 4. The starting point is a separation into a finite and a singular part,

$$
\mathrm{d} \sigma^{\gamma \gamma \rightarrow 4 f \gamma}=\mathrm{d} \sigma_{\text {finite }}^{\gamma \gamma \rightarrow 4 f \gamma}+\mathrm{d} \sigma_{\text {sing }}^{\gamma \gamma \rightarrow 4 f \gamma}
$$

where the soft and collinear singularities appear in $\mathrm{d} \sigma_{\text {sing }}^{\gamma \gamma \rightarrow 4 f \gamma}$ as $\ln \lambda$ and $\ln m_{f}$ terms, respectively.

The virtual corrections to the process (2.1) are calculated in the DPA, which is explained in Section 3. Since the real corrections are based on complete $\gamma \gamma \rightarrow 4 f \gamma$ matrix elements (i.e. they are not calculated in DPA), the cancellation of soft and collinear singularities in Eq. (2.5) requires particular care. To this end, we apply the DPA only to the finite part of the virtual corrections,

$$
\mathrm{d} \sigma_{\text {virt }}^{\gamma \gamma \rightarrow 4 f} \rightarrow \mathrm{d} \sigma_{\text {virt,finite,DPA }}^{\gamma \gamma \rightarrow \text { WW } \rightarrow 4 f}+\mathrm{d} \sigma_{\text {virt,sing }}^{\gamma \gamma \rightarrow 4 f} .
$$


Technically this is achieved by subtracting the singular part in DPA from the DPA virtual corrections and adding the exact singular part $\mathrm{d} \sigma_{\text {virt,sing }}^{\gamma \gamma \rightarrow 4 f}$. Of course, this procedure involves some freedom, because finite terms can be shifted between $\mathrm{d} \sigma_{\text {virt,finite,DPA }}^{\gamma \gamma \rightarrow 4 f}$ and $\mathrm{d} \sigma_{\text {virt,sing. }}^{\gamma \gamma \rightarrow 4 f}$. This arbitrariness is, however, of the order of the uncertainty $\mathcal{O}\left(\alpha \Gamma_{\mathrm{W}} /\left(\pi M_{\mathrm{W}}\right)\right)$ of our calculation. In the $\mathrm{e}^{+} \mathrm{e}^{-}$case this has been checked numerically in Ref. [10].

Inserting these rearrangements into Eq. (2.5) we obtain

$$
\int \mathrm{d} \sigma=\int \mathrm{d} \sigma_{\text {Born }}^{\gamma \gamma \rightarrow 4 f}+\int \mathrm{d} \sigma_{\text {virt,finite,DPA }}^{\gamma \gamma \rightarrow \mathrm{WW} \rightarrow 4 f}+\int \mathrm{d} \sigma_{\text {virt+real,sing }}^{\gamma \gamma \rightarrow 4 f}+\int \mathrm{d} \sigma_{\text {finite }}^{\gamma \gamma \rightarrow 4 f \gamma},
$$

where $\int \mathrm{d} \sigma_{\text {virt+real,sing }}^{\gamma \gamma \rightarrow 4 f}=\int \mathrm{d} \sigma_{\text {virt,sing }}^{\gamma \gamma \rightarrow 4 f}+\int \mathrm{d} \sigma_{\text {real,sing }}^{\gamma \gamma \rightarrow 4 f \gamma}$ does not contain any dependence on the photon mass anymore. Collinear singularities, appearing as $\ln m_{f}$ terms, also cancel if the observable is sufficiently inclusive. Such collinear-safe observables result if photons within cones collinear to any outgoing charged fermion are treated inclusively, i.e. if they are not separated from the nearly collinear fermion by any phase-space or event selection cuts. For non-collinear-safe observables logarithms of the fermion masses remain in the final result. This case demands a special treatment of the singular terms. We elaborate more on this issue in Section 4.2.

The radiative corrections are implemented in a Monte Carlo generator called COFFER $\gamma \gamma$, which is based on the lowest-order calculation described in Ref. [3]. We emphasize that we have actually constructed two independent Monte Carlo programs, each of which employs independent routines for the matrix elements (with and without corrections), for the subtraction procedure, and for the phase-space integration. The numerical results obtained with the two programs are in mutual agreement within statistical uncertainties.

\section{Virtual corrections}

\subsection{Concept of the double-pole approximation}

In the DPA the matrix element for $\gamma \gamma \rightarrow 4 f$ is expanded around the poles of the two resonant $\mathrm{W}$ propagators. The leading term of this expansion receives contributions from so-called factorizable and non-factorizable corrections. For the details of this classification, especially how a gauge-invariant decomposition is obtained, we refer to Refs. [10,11,20,21].

The generic Feynman diagram for the factorizable corrections is shown in Figure 1. It factorizes into the on-shell $\mathrm{W}$-pair production, the off-shell W-boson propagators, and the subsequent on-shell $\mathrm{W}$ decays. The corrections can be attributed to either of these subprocesses. When integrating over the full $4 f$ phase space, the $\mathrm{W}$ bosons usually are not on shell. However, a gauge-independent evaluation of the matrix elements for production and decay requires on-shell momenta for the $\mathrm{W}$ bosons. Therefore, we have to perform an on-shell projection, i.e. the momenta of the fermions are deformed in such a way that the W bosons become on shell. The deformation involves a certain freedom and introduces an error of $\mathcal{O}\left(\alpha \Gamma_{\mathrm{W}} /\left(\pi M_{\mathrm{W}}\right)\right)$. We define the on-shell projection by fixing the directions of the $\mathrm{W}^{+}$boson and of the fermions $f_{1}$ and $f_{3}$. The explicit formulas can be found in Appendix A of Ref. [10]. For later use, we label the new momenta $\hat{k}_{ \pm}$and $\hat{p}_{i}$ and define the kinematic invariants

$$
\hat{t}=\left(k_{1}-\hat{k}_{+}\right)^{2}=\left(k_{1}-\hat{p}_{1}-\hat{p}_{2}\right)^{2}, \quad \hat{u}=2 M_{\mathrm{W}}^{2}-s-\hat{t} .
$$




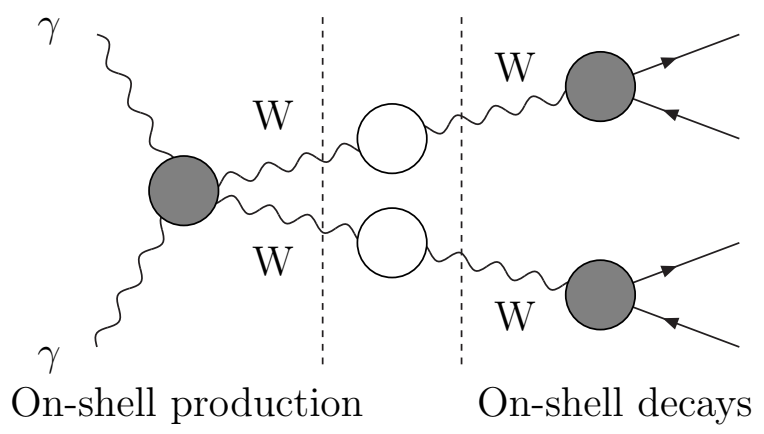

Figure 1: Generic Feynman diagram of the virtual factorizable corrections to $\gamma \gamma \rightarrow$ $\mathrm{WW} \rightarrow 4 f$. The shaded blobs stand for loop corrections to the production and decay processes.

Apart from the factorizable corrections there are additional doubly-resonant contributions. In the corresponding diagrams subprocesses are linked by a photon. These diagrams become doubly resonant in the limit of vanishing photon momentum, as can be seen from the soft-photon approximation in which the correction is proportional to the lowest-order cross section. The relative correction factor for these so-called non-factorizable corrections is, thus, not dependent of the actual production mechanism of the $\mathrm{W}$ pairs, but only on the electric charges and kinematics of the external particles of the process. The non-factorizable corrections were calculated in Refs. [20,21] for $\mathrm{e}^{+} \mathrm{e}^{-} \rightarrow \mathrm{WW} \rightarrow 4 f$. We can transfer the results for the $\mathrm{e}^{+} \mathrm{e}^{-}$case by simply omitting all contributions in which the exchanged photon is linked to an $\mathrm{e}^{ \pm}$from the initial state. The different types of relevant diagrams are depicted in Figure 2. The first two diagrams, labelled (a) and (b), are manifestly non-factorizable, i.e. the photon links different subprocesses so that the propagators in the diagrams cannot be factorized anymore. The diagrams (c), (d), and (e) contain both factorizable and non-factorizable contributions. Their factorizable parts are defined as the residues for on-shell $\mathrm{W}$ bosons times the off-shell W-boson propagators; note that this procedure introduces artificial soft IR divergences connected with the on-shellness of the $\mathrm{W}$ bosons in the loops. The non-factorizable parts of the diagrams are obtained from the difference of the doubly-resonant contribution of the full diagrams and their factorizable parts; the artificially introduced IR divergences of the factorizable parts are, thus, compensated by corresponding terms in the non-factorizable parts.

Following this strategy, the virtual corrections in DPA can be written as

$$
\begin{aligned}
\mathrm{d} \sigma_{\text {virt,DPA }}^{\gamma \gamma \rightarrow \mathrm{WW} \rightarrow 4 f}=\frac{1}{2 s} \int \mathrm{d} \Phi_{4 f}( & 2 \operatorname{Re}\left\{\delta \mathcal{M}_{\text {virt,fact }} \mathcal{M}_{\text {Born,DPA }}^{*}\right\} \\
& \left.+\delta_{\text {virt,nfact }}\left|\mathcal{M}_{\text {Born,DPA }}\right|^{2}+\left|\delta \mathcal{M}_{\text {Higgs }}\right|^{2}\right)
\end{aligned}
$$

where $\mathcal{M}_{\text {Born,DPA }}$ denotes the tree-level matrix element in DPA and $\delta_{\text {virt,nfact }}$ contains the non-factorizable corrections. The factorizable corrections $\delta \mathcal{M}_{\text {virt,fact }}$ also contain a contribution of the $s$-channel Higgs resonance, $\delta \mathcal{M}_{\text {Higgs }}$. In order to describe this resonance properly, it is not sufficient to include the interference of $\delta \mathcal{M}_{\text {Higgs }}$ with the Born matrix element, but the square of this matrix-element contribution has to be taken into account in 
addition. To this end, $\delta \mathcal{M}_{\text {Higgs }}$ has to be defined in a gauge-invariant way. Our treatment of $\delta \mathcal{M}_{\mathrm{Higgs}}$ is described in Section 3.2.4 in detail.

\subsection{Factorizable corrections}

\subsubsection{Calculation of the one-loop amplitudes}

The factorizable corrections comprise the corrections to the on-shell production of the $\mathrm{W}$ bosons and their on-shell decay and can be expressed as

$$
\begin{aligned}
& \delta \mathcal{M}_{\text {virt,fact }}=\sum_{\lambda_{+}, \lambda_{-}} \frac{1}{K_{+} K_{-}}\left(\delta \mathcal{M}^{\gamma \gamma \mathrm{W}^{+} \mathrm{W}^{-}} \mathcal{M}_{\mathrm{Born}}^{\mathrm{W}^{+} \rightarrow f_{1} \bar{f}_{2}} \mathcal{M}_{\mathrm{Born}}^{\mathrm{W}^{-} \rightarrow f_{3} \bar{f}_{4}}\right. \\
& +\mathcal{M}_{\mathrm{Born}}^{\gamma \gamma \mathrm{W}^{+} \mathrm{W}^{-}} \delta \mathcal{M}^{\mathrm{W}^{+} \rightarrow f_{1} \bar{f}_{2}} \mathcal{M}_{\mathrm{Born}}^{\mathrm{W}^{-} \rightarrow f_{3} \bar{f}_{4}} \\
& \left.+\mathcal{M}_{\mathrm{Born}}^{\gamma \gamma \mathrm{W}^{+} \mathrm{W}^{-}} \mathcal{M}_{\mathrm{Born}}^{\mathrm{W}^{+} \rightarrow f_{1} \bar{f}_{2}} \delta \mathcal{M}^{\mathrm{W}^{-} \rightarrow f_{3} \bar{f}_{4}}\right),
\end{aligned}
$$

where we introduced the abbreviations

$$
K_{ \pm}=k_{ \pm}^{2}-M_{\mathrm{W}}^{2}+\mathrm{i} M_{\mathrm{W}} \Gamma_{\mathrm{W}}
$$

and $\delta \mathcal{M}$ denote one-loop matrix elements. Note that all matrix elements on the r.h.s. of Eq. (3.3) depend on the on-shell projected momenta, but the momenta in $K_{ \pm}$remain unchanged. The results for the different one-loop corrections are already known in the literature [15-17,19]. Combining them in Eq. (3.3) is, however, non-trivial since the polarizations of the $\mathrm{W}$ bosons have to be defined consistently in a common reference frame.

The one-loop corrections $\delta \mathcal{M}^{\mathrm{W} \rightarrow f_{i} \bar{f}_{j}}$ to the $\mathrm{W}$ decays are rather simple. In the massless limit they are proportional to the respective Born matrix elements $\mathcal{M}_{\mathrm{Born}}^{\mathrm{W} \rightarrow f_{i} \bar{f}_{j}}$,

$$
\delta \mathcal{M}^{\mathrm{W} \rightarrow f_{i} \bar{f}_{j}}\left(\lambda_{\mathrm{W}}, \hat{p}_{i}, \hat{p}_{j}\right)=\delta^{\mathrm{W} \rightarrow f_{i} \bar{f}_{j}} \mathcal{M}_{\mathrm{Born}}^{\mathrm{W} \rightarrow f_{i} \bar{f}_{j}}\left(\lambda_{\mathrm{W}}, \hat{p}_{i}, \hat{p}_{j}\right)
$$

where $\delta^{\mathrm{W} \rightarrow f_{i} \bar{f}_{j}}$ is a constant correction factor that neither depends on the kinematics nor on the helicity $\lambda_{\mathrm{W}}$ of the decaying $\mathrm{W}$ boson.

The one-loop correction $\delta \mathcal{M}^{\gamma \gamma \mathrm{W}^{+} \mathrm{W}^{-}}$to the $\mathrm{W}$-pair production process contains the complicated part; we have derived it in two independent ways. One calculation is based on the results of Ref. [16] which were obtained in a gauge with a non-linear gauge fixing term. The other is a new calculation based on the program FeynArts [24] for the generation of the amplitudes and on in-house Mathematica routines for their algebraic reduction. This second calculation has been carried out in the 't Hooft-Feynman gauge and repeated in the background-field gauge [25] to get an additional consistency check. The results obtained from the different calculations are in mutual numerical agreement.

In the following we describe an efficient way for calculating the contribution of $\delta \mathcal{M}^{\gamma \gamma \mathrm{W}^{+} \mathrm{W}^{-}}$to $\delta \mathcal{M}_{\text {virt,fact }}$ of Eq. (3.3), taking into account all spin correlations. As described in Ref. [16], the matrix element $\delta \mathcal{M}^{\gamma \gamma \mathrm{W}^{+} \mathrm{W}^{-}}$for on-shell $\mathrm{W}$-pair production is decomposed into a sum of products of form factors $F_{j}$, which only depend on the kine- 


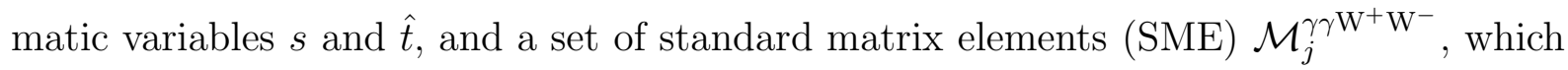
contain the polarizations and momenta of the external photons and $\mathrm{W}$ bosons,

$$
\begin{aligned}
& \delta \mathcal{M}^{\gamma \gamma \mathrm{W}^{+} \mathrm{W}^{-}}\left(k_{1}, k_{2}, \lambda_{1}, \lambda_{2} ; \hat{k}_{+}, \hat{k}_{-}, \lambda_{+}, \lambda_{-}\right) \\
& =\sum_{j=1}^{36} F_{j}(s, \hat{t}) \mathcal{M}_{j}^{\gamma \gamma \mathrm{W}^{+} \mathrm{W}^{-}}\left(k_{1}, k_{2}, \lambda_{1}, \lambda_{2} ; \hat{k}_{+}, \hat{k}_{-}, \lambda_{+}, \lambda_{-}\right) .
\end{aligned}
$$

The SME $\mathcal{M}_{j}^{\gamma \gamma \mathrm{W}^{+} \mathrm{W}^{-}}$are obtained from the 83 basic matrix elements given in Section 2 of Ref. [16] which are reduced to 36 matrix elements as described there ${ }^{2}$. The decay matrix elements $\mathcal{M}_{\mathrm{Born}}^{\mathrm{W} \rightarrow f_{i} \bar{f}_{j}}$, which multiply $\delta \mathcal{M}^{\gamma \gamma \mathrm{W}^{+} \mathrm{W}^{-}}$in Eq. (3.3), can be included by replacing the $\mathrm{W}$ polarization vectors $\varepsilon_{ \pm}^{*}$ in the definitions of the $\mathrm{SME} \mathcal{M}_{j}$ by the "effective polarization vectors"

$$
\hat{\varepsilon}_{+}^{* \mu}=\frac{e}{\sqrt{2} s_{\mathrm{w}}} \frac{1}{K_{+}} \bar{u}\left(\hat{p}_{1}\right) \gamma^{\mu} \omega_{-} v\left(\hat{p}_{2}\right), \quad \hat{\varepsilon}_{-}^{* \mu}=\frac{e}{\sqrt{2} s_{\mathrm{w}}} \frac{1}{K_{-}} \bar{u}\left(\hat{p}_{3}\right) \gamma^{\mu} \omega_{-} v\left(\hat{p}_{4}\right),
$$

where $\bar{u}\left(\hat{p}_{i}\right)$ and $v\left(\hat{p}_{i}\right)$ are the Dirac spinors of the fermions and antifermions and $\omega_{-}=$ $\frac{1}{2}\left(1-\gamma_{5}\right)$ is the left-handed chirality projector. The effective $\mathrm{W}$-polarization vectors $\hat{\varepsilon}_{ \pm}^{*}$ are formal shorthands for the $\mathrm{W}$ propagators and the tree-level decay matrix elements, which involve the usual $\mathrm{SU}(2)$ gauge coupling $e / s_{\mathrm{w}}$. Upon substituting $\varepsilon_{ \pm}^{*} \rightarrow \hat{\varepsilon}_{ \pm}^{*}$ in the SME for on-shell W-pair production, we obtain a new set of SME $\mathcal{M}_{j}$ that correctly transfer the W polarization to the decay,

$$
\begin{aligned}
\mathcal{M}_{j}\left(k_{1}, k_{2}, \lambda_{1}, \lambda_{2} ; k_{+}^{2}, k_{-}^{2} ;\left\{\hat{p}_{i}\right\}\right)= & \left.\mathcal{M}_{j}^{\gamma \gamma \mathrm{W}^{+} \mathrm{W}^{-}}\left(k_{1}, k_{2}, \lambda_{1}, \lambda_{2} ; \hat{k}_{+}, \hat{k}_{-}, \lambda_{+}, \lambda_{-}\right)\right|_{\varepsilon_{ \pm}^{*} \rightarrow \hat{\varepsilon}_{ \pm}^{*}} \\
= & \sum_{\lambda_{+}, \lambda_{-}} \frac{1}{K_{+} K_{-}} \mathcal{M}_{j}^{\gamma \gamma \mathrm{W}^{+} \mathrm{W}^{-}}\left(k_{1}, k_{2}, \lambda_{1}, \lambda_{2} ; \hat{k}_{+}, \hat{k}_{-}, \lambda_{+}, \lambda_{-}\right) \\
& \times \mathcal{M}_{\mathrm{Born}}^{\mathrm{W}^{+} \rightarrow f_{1} \bar{f}_{2}}\left(\lambda_{+}, \hat{p}_{1}, \hat{p}_{2}\right) \mathcal{M}_{\mathrm{Born}}^{\mathrm{W}^{-} \rightarrow f_{3} \bar{f}_{4}}\left(\lambda_{-}, \hat{p}_{3}, \hat{p}_{4}\right) .
\end{aligned}
$$

The new SME $\mathcal{M}_{j}$ can be easily evaluated with spinor methods, as e.g. described in Ref. [26].

In summary the factorizable part of the virtual correction takes the form

$$
\begin{aligned}
\delta \mathcal{M}_{\text {virt,fact }}= & \sum_{j=1}^{36} F_{j}(s, \hat{t}) \mathcal{M}_{j}\left(k_{1}, k_{2}, \lambda_{1}, \lambda_{2} ; k_{+}^{2}, k_{-}^{2} ;\left\{\hat{p}_{i}\right\}\right) \\
& +\left(\delta^{\mathrm{W}^{+} \rightarrow f_{1} \bar{f}_{2}}+\delta^{\mathrm{W}^{-} \rightarrow f_{3} \bar{f}_{4}}\right) \mathcal{M}_{\mathrm{Born}, \mathrm{DPA}}\left(k_{1}, k_{2}, \lambda_{1}, \lambda_{2} ; k_{+}^{2}, k_{-}^{2} ;\left\{\hat{p}_{i}\right\}\right) .
\end{aligned}
$$

\subsubsection{Details of the numerical evaluation}

The formulas for the coefficient functions $F_{j}$ are rather lengthy and contain many oneloop integrals, which in turn involve many dilogarithmic functions, etc. Thus, to speed up the numerical evaluation it is desirable not to evaluate the $F_{j}$ at each phase-space point. Moreover, numerical instabilities occur at the boundary of the phase space where

\footnotetext{
${ }^{2}$ The on-shell momenta $\hat{k}_{ \pm}$and the helicities $\lambda_{ \pm}$of the $\mathrm{W}$ bosons are denoted $k_{3,4}$ and $\lambda_{3,4}$ in Ref. [16].
} 
the scattering angle $\theta$ between the $\mathrm{W}$ bosons and the beam axis tends to 0 or $\pi$. This is due to the inverse Gram determinants appearing in the Passarino-Veltman reduction [27] of the tensor integrals. The problems of speed and stability can be solved by expanding the functions $F_{j}(s, \hat{t})$ in terms of a generalized Fourier series in the variable $\hat{t}$ for fixed values of $s$. The coefficients of this expansion are calculated before the Monte Carlo integration. An appropriate system of orthogonal functions in the variable $x=\cos \theta$, which is equivalent to a function of $\hat{t}$ for fixed $s$, is provided by the Legendre polynomials

$$
P_{l}(x)=\frac{1}{2^{l} l !} \frac{\mathrm{d}^{l}}{\mathrm{~d} x^{l}}\left[\left(x^{2}-1\right)^{l}\right], \quad l=0,1, \ldots .
$$

For this basis functions, the coefficients read

$$
c_{j, l}(s)=\frac{2 l+1}{2} \int_{-1}^{+1} \mathrm{~d} \cos \theta\left(\hat{t}-M_{\mathrm{W}}^{2}\right)\left(\hat{u}-M_{\mathrm{W}}^{2}\right) F_{j}(s, \hat{t}) P_{l}(\cos \theta),
$$

where we have introduced the factor $\left(\hat{t}-M_{\mathrm{W}}^{2}\right)\left(\hat{u}-M_{\mathrm{W}}^{2}\right)$ in order to flatten the $t$ - and $u$-channel poles in the functions $F_{j}$. This improves the efficiency of the expansion. The integration in Eq. (3.11) is carried out using Gaussian integration. With 40 integration points the region of instability is not entered (for energies up to a few $\mathrm{TeV}$ ), and the integration is sufficiently precise. During the Monte Carlo integration the coefficient functions are recovered by the generalized Fourier series

$$
F_{j}(s, \hat{t})=\sum_{l=0}^{\infty} \frac{1}{\left(\hat{t}-M_{\mathrm{W}}^{2}\right)\left(\hat{u}-M_{\mathrm{W}}^{2}\right)} c_{j, l}(s) P_{l}(\cos \theta) .
$$

In Ref. [10] the same concept was used to evaluate the factorizable corrections to $\mathrm{e}^{+} \mathrm{e}^{-} \rightarrow \mathrm{WW} \rightarrow 4 f$; there it was sufficient to use the Legendre polynomials up to $l=20$ for a good accuracy. In the case of $\gamma \gamma \rightarrow \mathrm{WW}$, however, the coefficient functions involve inverse Gram determinants $1 /\left(\hat{t} \hat{u}-M_{\mathrm{W}}^{4}\right) \propto 1 / \sin ^{2} \theta$ which appear in the PassarinoVeltman reduction of the tensor integrals. As each step in this recursive reduction involves such an inverse determinant, $1 /\left(\hat{t} \hat{u}-M_{\mathrm{W}}^{4}\right)$ can appear up to the fourth power. At $\cos \theta \approx$ \pm 1 this factor leads to a behaviour of the $F_{j}(s, \hat{t})$ that is not well approximated by the Legendre expansion. Using higher-order Legendre polynomials is not a solution since this increases the calculation time and also requires more integration points for the Gaussian integration. The more points are used in the Gaussian integration, the closer some of these points approach the integration boundary where the numerical stability of the coefficient function breaks down. Therefore, we follow a different strategy based on the fact that the helicity amplitudes for the on-shell process $\gamma \gamma \rightarrow \mathrm{WW}$ are smooth functions of $\cos \theta$, apart from the $t$ - and $u$-channel poles. Thus, within the full amplitude the factors $1 /\left(\hat{t} \hat{u}-M_{\mathrm{W}}^{4}\right)$ have to cancel between contributions of different coefficient functions. To make use of this fact we change the basis of SME by a linear transformation in such a way that the new coefficient functions correspond to helicity amplitudes of the on-shell process $\gamma \gamma \rightarrow$ WW. Some details of this transformation can be found in App. A. After this transformation the uncertainty of the approximated matrix elements in Eq. (3.6) is well below $10^{-4}$ with respect to the Born matrix elements for all values of $\cos \theta$.

In contrast to the $\mathrm{e}^{+} \mathrm{e}^{-}$case, the CM energy $\sqrt{s}$ of the photons is not fixed. Thus, we have to perform the Legendre expansions for different values of $s$. During the Monte Carlo 
integration we derive an approximate value of the coefficients $c_{j, l}(s)$ by interpolation. Since the $F_{j}(s, \hat{t})$ depend on $s$ very smoothly, it is sufficient to calculate the $c_{j, l}(s)$ at intervals of $\Delta s \lesssim 1 \mathrm{GeV}$. In these intervals we then interpolate with a polynomial of third order. We have checked that, up to $1 \mathrm{TeV}$, this yields a sufficient accuracy (i.e. better than the accuracy of the Legendre expansion).

\subsubsection{Renormalization and imaginary parts of virtual corrections}

For on-shell W-pair production, which was considered in Ref. [16], imaginary parts of counterterms, if included, do not influence the correction to the matrix element square. The reason is that for the $2 \rightarrow 2$ scattering process $\gamma \gamma \rightarrow \mathrm{WW}$ all SME, and thus also the Born matrix element, can be taken real by appropriate phase choices. Thus, the operation of taking the real part in the interference term $2 \operatorname{Re}\left\{\mathcal{M}_{\mathrm{ct}} \mathcal{M}_{\text {Born }}^{*}\right\}$ of the counterterm contribution $\mathcal{M}_{\text {ct }}$ to the one-loop amplitude with the Born amplitude effectively acts on the renormalization constants themselves. The same argument shows that also imaginary parts of loop integrals drop out. These arguments are no longer true if the decay of the W bosons is taken into account, because the SME and the Born matrix element $\mathcal{M}_{\text {Born,DPA }}$ become necessarily complex. Thus, imaginary parts of renormalization constants and of loop integrals in general matter. Considering the W-decay amplitudes in the DPA in more detail, as e.g. done in Ref. [13] for the $\mathrm{e}^{+} \mathrm{e}^{-}$case, one can see that imaginary parts average to zero after the azimuthal decay angles of the W-decay products are integrated over.

We have calculated the virtual corrections taking into account the imaginary parts of all loop integrals. As already mentioned, we carried out the whole loop calculation in different gauges: in the 't Hooft-Feynman gauge [28], in a non-linear gauge [16], and in the background-field gauge [25]. We find agreement between the results obtained in these different gauges, but only if we also take into account the imaginary parts of the loops that contribute to renormalization constants. In order to explain this fact, we consider the counterterm contributions to the one-loop matrix element in more detail.

Following Ref. [16], we write the Born matrix element in DPA as

$$
\mathcal{M}_{\mathrm{Born}, \mathrm{DPA}}=8 \pi \alpha\left\{\frac{s}{M_{\mathrm{W}}^{2}-\hat{t}} \mathcal{M}_{0, t}+\frac{s}{M_{\mathrm{W}}^{2}-\hat{u}} \mathcal{M}_{0, u}-\left(\varepsilon_{1} \varepsilon_{2}\right)\left(\hat{\varepsilon}_{+}^{*} \hat{\varepsilon}_{-}^{*}\right)\right\},
$$

where $\mathcal{M}_{0, t}$ and $\mathcal{M}_{0, u}$ are abbreviations for specific combinations of momenta and polarization vectors defined as in Eq. (22) of Ref. [16] for on-shell W-pair production. In the 't Hooft-Feynman gauge, the counterterm contribution to the production part of the factorizable correction reads

$$
\begin{aligned}
\delta \mathcal{M}_{\mathrm{ct}, \mathrm{prod}}^{\mathrm{tHF}}= & \mathcal{M}_{\mathrm{Born}, \mathrm{DPA}}\left(2 \delta Z_{e}+\delta Z_{W}+\delta Z_{A A}-\frac{c_{\mathrm{w}}}{s_{\mathrm{w}}} \delta Z_{Z A}\right) \\
& -8 \pi \alpha\left(\frac{s \delta M_{\mathrm{W}}^{2}}{\left(\hat{t}-M_{\mathrm{W}}^{2}\right)^{2}} \mathcal{M}_{0, t}+\frac{s \delta M_{\mathrm{W}}^{2}}{\left(\hat{u}-M_{\mathrm{W}}^{2}\right)^{2}} \mathcal{M}_{0, u}\right) \\
& -4 \pi \alpha\left(\frac{\left(\varepsilon_{1} \hat{\varepsilon}_{+}^{*}\right)\left(\varepsilon_{2} \hat{\varepsilon}_{-}^{*}\right)}{\left(\hat{t}-M_{\mathrm{W}}^{2}\right)}+\frac{\left(\varepsilon_{1} \hat{\varepsilon}_{-}^{*}\right)\left(\varepsilon_{2} \hat{\varepsilon}_{+}^{*}\right)}{\left(\hat{u}-M_{\mathrm{W}}^{2}\right)}\right)\left(2 \delta M_{\mathrm{W}}^{2}+\frac{M_{\mathrm{W}}^{2}}{s_{\mathrm{W}} c_{\mathrm{w}}} \delta Z_{Z A}\right) \\
& +4 \pi \alpha \frac{e M_{\mathrm{W}}}{2 s_{\mathrm{w}}}\left(\frac{\left(\varepsilon_{1} \hat{\varepsilon}_{+}^{*}\right)\left(\varepsilon_{2} \hat{\varepsilon}_{-}^{*}\right)}{\left(\hat{t}-M_{\mathrm{W}}^{2}\right)^{2}}+\frac{\left(\varepsilon_{1} \hat{\varepsilon}_{-}^{*}\right)\left(\varepsilon_{2} \hat{\varepsilon}_{+}^{*}\right)}{\left(\hat{u}-M_{\mathrm{W}}^{2}\right)^{2}}\right) \delta t
\end{aligned}
$$


where we adopt the conventions of Ref. [28] for the renormalization constants $\delta Z_{e}, \delta Z_{W}$, etc. The explicit calculation of the constants in terms of self-energies is also described there. The counterterm contribution in the background-field gauge [25] can be obtained from $\delta \mathcal{M}_{\text {ct,prod }}^{\mathrm{tHF}}$ by simply omitting the $\delta Z_{Z A}$ terms, because $\delta Z_{Z A}$ vanishes owing to the background-field gauge invariance. In the non-linear gauge the counterterm contribution reads

$$
\begin{aligned}
\delta \mathcal{M}_{\mathrm{ct}, \text { prod }}^{\mathrm{NL}}= & \mathcal{M}_{\mathrm{Born}, \mathrm{DPA}}\left(2 \delta Z_{e}+\delta Z_{W}+\delta Z_{A A}-\frac{c_{\mathrm{w}}}{s_{\mathrm{w}}} \delta Z_{Z A}\right) \\
& -8 \pi \alpha\left(\frac{s \delta M_{\mathrm{W}}^{2}}{\left(\hat{t}-M_{\mathrm{W}}^{2}\right)^{2}} \mathcal{M}_{0, t}+\frac{s \delta M_{\mathrm{W}}^{2}}{\left(\hat{u}-M_{\mathrm{W}}^{2}\right)^{2}} \mathcal{M}_{0, u}\right)
\end{aligned}
$$

as described in Ref. [16], which is different from its counterpart in 't Hooft-Feynman gauge. Note also that the explicit expressions of the renormalization constants in the different gauges are in general different.

Imaginary parts of loop and counterterm contributions that are proportional to the Born matrix element, $\delta \mathcal{M}=c \mathcal{M}_{\text {Born }}$, cannot influence matrix element squares, because $2 \operatorname{Re}\left\{\delta \mathcal{M M}_{\text {Born }}^{*}\right\}=2 \operatorname{Re}\{c\}\left|\mathcal{M}_{\text {Born }}\right|^{2}$. Thus, the $\mathrm{W}$-mass renormalization constant $\delta M_{\mathrm{W}}^{2}$ is the only renormalization constant whose imaginary part plays a role, since the tadpole counterterm $\delta t$ is a real quantity. From Eqs. (3.14) and (3.15), we see that $\delta M_{\mathrm{W}}^{2}$, which is equal in all three considered gauges, enters the counterterm contributions in the "t HooftFeynman gauge and in the non-linear gauge in different ways. In fact, we have checked numerically that the virtual corrections in these two gauges are different (though finite) if the usual on-shell prescription $\delta M_{\mathrm{W}}^{2}=\operatorname{Re}\left\{\Sigma_{\mathrm{T}}^{W}\left(M_{\mathrm{W}}^{2}\right)\right\}$ (see e.g. Ref. [28]) is applied, where $\Sigma_{\mathrm{T}}^{W}\left(k^{2}\right)$ is the transverse part of the W-boson self-energy with momentum transfer $k$. If we, on the other hand, use the definition $\delta M_{\mathrm{W}}^{2}=\Sigma_{\mathrm{T}}^{W}\left(M_{\mathrm{W}}^{2}\right)$, i.e. without taking the real part of the self-energy, we find agreement for the results from the different gauges. This clearly shows that the imaginary part of a one-loop amplitude is in general gauge dependent if imaginary parts in renormalization constants are not taken into account. The reason for this fact, in other words, is that the decomposition of a renormalized transition matrix element into genuine loop parts and counterterm contributions depends on the gauge fixing. ${ }^{3}$

In our Monte Carlo generator we have taken into account the imaginary parts of the virtual corrections (including the ones from counterterms); more precisely they can be switched on and off optionally. As explained above, they could only affect observables that are sensitive to the azimuthal decay angles of the fermions. In our numerical results, we could, however, find no significant effects.

\subsubsection{Higgs resonance}

The loop-induced Higgs resonance, $\gamma \gamma \rightarrow \mathrm{H} \rightarrow \mathrm{WW} \rightarrow 4 f$, belongs to the class of factorizable contributions. Nevertheless, its treatment, especially the question of gauge invariance when including the Higgs decay width, deserves some care. In Ref. [16] the

\footnotetext{
${ }^{3} \mathrm{~A}$ consistent renormalization prescription with complex renormalization constants naturally leads to complex masses for unstable particles. Such a renormalization scheme was proposed recently in Ref. [8] in the context of a full $\mathcal{O}(\alpha)$ calculation for $\mathrm{e}^{+} \mathrm{e}^{-} \rightarrow 4 f$.
} 
diagrams with an $s$-channel Higgs resonance were decomposed into a gauge-invariant resonant part and a gauge-dependent non-resonant part. If we write the contribution of the Higgs-exchange diagrams as

$$
\delta \mathcal{M}^{\gamma \gamma \mathrm{H}}=\frac{F^{H}(s)}{s-M_{\mathrm{H}}^{2}}\left(\varepsilon_{1} \varepsilon_{2}\right)\left(\hat{\varepsilon}_{+}^{*} \hat{\varepsilon}_{-}^{*}\right)
$$

with $F^{H}(s)$ given in Section 4.3 of Ref. [16], and $\varepsilon_{1}$ and $\varepsilon_{2}$ being the polarization vectors of the photons, then the Higgs decay width can be introduced by replacing

$$
\delta \mathcal{M}^{\gamma \gamma \mathrm{H}} \rightarrow\left(\frac{F^{H}\left(M_{\mathrm{H}}^{2}\right)}{s-M_{\mathrm{H}}^{2}+\mathrm{i} M_{\mathrm{H}} \Gamma_{\mathrm{H}}}+\frac{F^{H}(s)-F^{H}\left(M_{\mathrm{H}}^{2}\right)}{s-M_{\mathrm{H}}^{2}}\right)\left(\varepsilon_{1} \varepsilon_{2}\right)\left(\hat{\varepsilon}_{+}^{*} \hat{\varepsilon}_{-}^{*}\right) .
$$

As the residue $F^{H}\left(M_{\mathrm{H}}^{2}\right)$ is gauge independent, we have introduced the Higgs decay width $\Gamma_{\mathrm{H}}$ in a gauge-invariant way. Recall that the choice of the polarization vectors of the photons is such that they obey

$$
\varepsilon_{i} k_{j}=0, \quad i, j=1,2 .
$$

Close to the resonance, the contribution of the Higgs-exchange diagrams is strongly enhanced. This is why we also take into account the square of the resonant part in Eq. (3.2),

$$
\delta \mathcal{M}_{\text {Higgs }}=\frac{F^{H}\left(M_{\mathrm{H}}^{2}\right)\left(\varepsilon_{1} \varepsilon_{2}\right)\left(\hat{\varepsilon}_{+}^{*} \hat{\varepsilon}_{-}^{*}\right)}{s-M_{\mathrm{H}}^{2}+\mathrm{i} M_{\mathrm{H}} \Gamma_{\mathrm{H}}} .
$$

In this approach only the leading contribution to the Higgs resonance is taken into account. However, the gauge-invariant separation of $\delta \mathcal{M}_{\text {Higgs }}$ from the remaining one-loop amplitude easily allows for specific improvements in predictions for the Higgs-production signal in the future. To this end, a pole expansion about the Higgs resonance would be an adequate first step. Conceptually this expansion again leads to factorizable and non-factorizable contributions, but the corresponding ingredients are not all available yet and their calculation is beyond the scope of this work. It should be mentioned that both the $\mathcal{O}(\alpha)$ electroweak and $\mathcal{O}\left(\alpha_{\mathrm{s}}\right)$ QCD virtual factorizable corrections to (on-shell) Higgs production $\gamma \gamma \rightarrow \mathrm{H}$ can be deduced from the corresponding two-loop calculations [29] (see also references therein) for the decay $\mathrm{H} \rightarrow \gamma \gamma$.

\subsection{Non-factorizable corrections}

As explained in Section 3.1, we make use of the result for the non-factorizable corrections to $\mathrm{e}^{+} \mathrm{e}^{-} \rightarrow \mathrm{WW} \rightarrow 4 f$. According to Refs. [10,21] we write the correction factor

to the lowest-order cross section as a sum over contributions that are associated with different pairs of fermions,

$$
\delta_{\text {virt,nfact }}=\sum_{a=1,2} \sum_{b=3,4}(-1)^{a+b+1} Q_{a} Q_{b} \frac{\alpha}{\pi} \operatorname{Re}\left\{\Delta^{\mathrm{virt}}\left(k_{+}, p_{a} ; k_{-}, p_{b}\right)\right\} .
$$

The function $\Delta^{\text {virt }}$ receives contributions from the different types of diagrams in Figure 2,

$$
\Delta^{\mathrm{virt}}=\Delta_{\mathrm{mf}^{\prime}}^{\mathrm{virt}}+\Delta_{\mathrm{ff}^{\prime}}^{\mathrm{virt}}+\Delta_{\mathrm{mm}^{\prime}}^{\mathrm{virt}}+\Delta_{\mathrm{mf}}^{\mathrm{virt}}+\Delta_{\mathrm{mm}}^{\mathrm{virt}}
$$


(a) type $\left(\mathrm{mf}^{\prime}\right)$

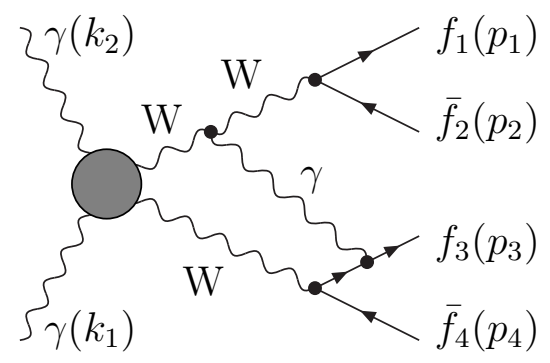

(c) type $\left(\mathrm{mm}^{\prime}\right)$

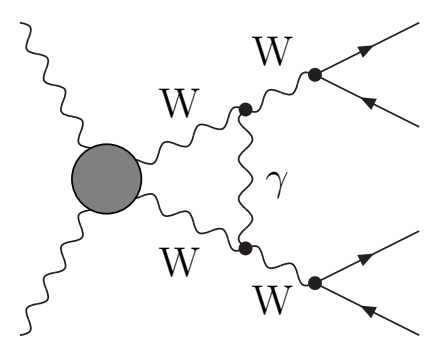

(b) type $\left(\mathrm{ff}^{\prime}\right)$

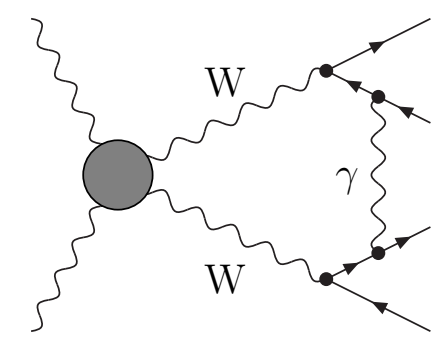

(d) type (mf)

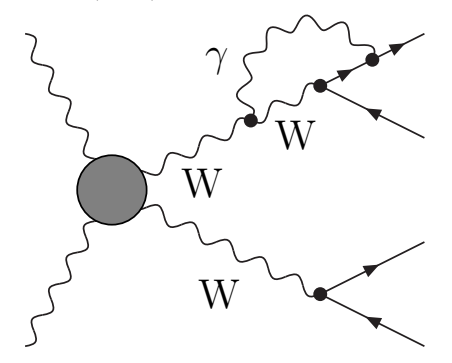

(e) type $(\mathrm{mm})$

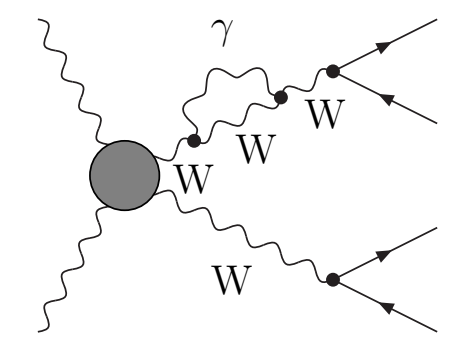

Figure 2: A representative set of diagrams contributing to the virtual non-factorizable corrections. The shaded blobs stand for all tree-level structures contributing to $\gamma \gamma \rightarrow$ WW.

for which the results were given in terms of scalar integrals in Ref. [10]. The final result for $a=2, b=3$ (all other contributions can be derived by appropriate substitutions) is

$$
\begin{aligned}
\Delta_{\mathrm{mf}}^{\mathrm{virt}}+ & \Delta_{\mathrm{ff}^{\prime}}^{\mathrm{virt}}+\Delta_{\mathrm{mf}}^{\mathrm{virt}} \\
\sim & -\frac{K_{+} K_{-} s_{23} \operatorname{det}\left(Y_{0}\right)}{\operatorname{det}(Y)} D_{0}\left(-p_{4}, k_{+}+p_{3}, p_{2}+p_{3}, 0, M, M, 0\right) \\
& -\frac{K_{+} \operatorname{det}\left(Y_{3}\right)}{\operatorname{det}(Y)} F_{3}-\frac{K_{-} \operatorname{det}\left(Y_{2}\right)}{\operatorname{det}(Y)} F_{2}+\ln \left(\frac{\lambda^{2}}{M_{\mathrm{W}}^{2}}\right) \ln \left(-\frac{s_{23}}{M_{\mathrm{W}}^{2}}-\mathrm{i} \epsilon\right), \\
\Delta_{\mathrm{mm}}^{\mathrm{virt}} \sim & \left(2 M_{\mathrm{W}}^{2}-s\right)\left\{C_{0}\left(k_{+},-k_{-}, 0, M, M\right)-\left.C_{0}\left(k_{+},-k_{-}, \lambda, M_{\mathrm{W}}, M_{\mathrm{W}}\right)\right|_{k_{ \pm}^{2}=M_{\mathrm{W}}^{2}}\right\}, \\
\Delta_{\mathrm{mm}}^{\mathrm{virt}} \sim & 2 \ln \left(\frac{\lambda M_{\mathrm{W}}}{-K_{+}}\right)+2 \ln \left(\frac{\lambda M_{\mathrm{W}}}{-K_{-}}\right)+4
\end{aligned}
$$


where the sign " " indicates that the limit $k_{ \pm}^{2} \rightarrow M_{\mathrm{W}}^{2}$ and $\Gamma_{\mathrm{W}} \rightarrow 0$ is carried out whenever this does not lead to a singularity. The matrices $Y_{0}, Y_{2}, Y_{3}$, and $Y$ arise from the reduction of 5-point functions and can be found in Section 3.1 of Ref. [21]. The functions $F_{2}$ and $F_{3}$ are defined in Section 4.2, and the $C_{0}$ and $D_{0}$ functions in Appendix C.1 of the same reference. The contribution $\Delta_{\mathrm{mm}^{\prime}}^{\text {virt }}$ contains the difference of the full off-shell and on-shell Coulomb singularity, as described there in detail.

The full correction factor $\delta_{\text {virt,nfact }}$ does not contain fermion-mass singularities [10], but involves IR-singular terms $\ln \lambda$, as explicitly visible in Eq. (3.22). The latter originate from the subtraction of the virtual factorizable correction, which involves the one-loop matrix elements for $\gamma \gamma \rightarrow \mathrm{WW}$ and $\mathrm{W} \rightarrow f \bar{f}^{\prime}$ with on-shell $\mathrm{W}$ bosons, from the doubly-resonant part of the matrix element for the full $\gamma \gamma \rightarrow 4 f$ process. Specifically, the $\ln \lambda$ terms stem from diagrams with photon exchange between an on-shell $\mathrm{W}$ boson and another on-shell particle. As already explained in Section 3.1, these singularities cancel in the sum of factorizable and non-factorizable contributions, since they are artificially introduced in the corresponding decomposition of the virtual correction.

\subsection{Leading universal corrections and input-parameter scheme}

We parametrize the cross section in such a way that the universal corrections arising from the running of the electromagnetic coupling $\alpha$ and from the $\rho$-parameter are absorbed in the lowest order. To this end, we take all particle masses as input, from which the weak mixing angle is derived via the on-shell condition

$$
s_{\mathrm{w}}^{2}=1-c_{\mathrm{w}}^{2}=1-\frac{M_{\mathrm{W}}^{2}}{M_{\mathrm{Z}}^{2}} .
$$

The electromagnetic coupling $\alpha$ is chosen in order to absorb some universal corrections.

As pointed out in Ref. [16], the relevant coupling for the $\gamma \gamma \rightarrow$ WW production process is the fine-structure constant $\alpha(0)$, because the external on-shell photons do not induce any running in their coupling to the $\mathrm{W}$ bosons. This means that the on-shell renormalization is carried out precisely as described in Refs. [25,28] for this contribution.

For the decay of the $\mathrm{W}$ bosons, it is, however, appropriate to derive $\alpha$ from the Fermi constant $G_{\mu}$ leading to

$$
\alpha_{G_{\mu}}=\frac{\sqrt{2} G_{\mu} M_{\mathrm{W}}^{2} s_{\mathrm{w}}^{2}}{\pi}
$$

This modification of the coupling induces an additional finite contribution to the charge renormalization constant,

$$
\left.\delta Z_{\mathrm{e}}\right|_{G_{\mu}}=\left.\delta Z_{\mathrm{e}}\right|_{\alpha(0)}-\frac{1}{2} \Delta r
$$

where $\left.\delta Z_{\mathrm{e}}\right|_{\alpha(0)}$ is the charge renormalization constant of the on-shell renormalization schemes [25,28] with $\alpha(0)$ as renormalized coupling. The quantity $\Delta r$ contains the radiative corrections to muon decay; explicit expressions for $\Delta r$ can, e.g., be found in Refs. [28,30].

In summary, our lowest-order cross section scales like $\alpha(0)^{2} \alpha_{G_{\mu}}^{2}$. For the relative $\mathcal{O}(\alpha)$ corrections we use $\alpha(0)$, which is the correct effective coupling for real photon emission, so

that the corrected cross section scales like $\alpha(0)^{3} \alpha_{G_{\mu}}^{2}$. For the loop-induced Higgs resonance 
we exceptionally take the scaling factor $\alpha(0)^{2} \alpha_{G_{\mu}}^{3}$, which accounts for the two "photonic" and the three "weak" couplings in the corresponding diagrams. We perform this rescaling, of course, only in the gauge-invariant resonant part $\delta \mathcal{M}_{\text {Higgs }}$ of the one-loop amplitude, as defined in Eq. (3.19).

\subsection{Improved Born approximation}

The motivation for calculating the virtual corrections in DPA lies in the dominance of doubly-resonant diagrams. At threshold, however, singly-resonant and nonresonant diagrams become equally important, thus, rendering the naive error estimate of $\mathcal{O}\left(\alpha \Gamma_{\mathrm{W}} /\left(M_{\mathrm{W}} \pi\right)\right)$ unreliable. As a consequence, we decided to use the DPA only for a CM energy $\sqrt{s_{\gamma \gamma}}>170 \mathrm{GeV}$ when integrating over the photon spectrum. For $\sqrt{s_{\gamma \gamma}}<170 \mathrm{GeV}$ we make use of an improved Born approximation (IBA), i.e. we approximate the $\mathcal{O}(\alpha)$ corrections by universal contributions without any expansion about the $\mathrm{W}$ resonances. Assuming that the IBA accounts for all $\mathcal{O}(\alpha)$ corrections with pronounced enhancement factors, the relative uncertainty of the IBA is about $\sim \pm 2 \%$. For the corresponding $\mathrm{e}^{+} \mathrm{e}^{-}$reaction this expectation was confirmed by the full $\mathcal{O}(\alpha)$ calculation $[7,8]$ for $4 f$ production.

In detail, we start from the Born cross section based on the full set of $\gamma \gamma \rightarrow 4 f$ diagrams, which is parametrized as described in the previous section and include the Higgs resonance with SM couplings. Such lowest-order predictions, which we denote "Born+Higgs" below, have already been presented in Ref. [3]. In addition, we now dress the resulting cross section with the off-shell Coulomb singularity,

$$
\int \mathrm{d} \sigma_{\text {IBA }}^{\gamma \gamma \rightarrow 4 f}=\frac{1}{2 s} \int \mathrm{d} \Phi_{4 f}\left(1+\delta_{\text {coul }}\right)\left|\mathcal{M}_{\text {Born+Higgs }}^{\gamma \gamma \rightarrow 4 f}\right|^{2} .
$$

The correction factor $\delta_{\text {coul }}$ for the Coulomb singularity was calculated in Ref. [31] to

$$
\delta_{\text {coul }}=\frac{\alpha(0)}{\bar{\beta}} \operatorname{Im}\left\{\ln \left(\frac{\beta+\Delta-\bar{\beta}}{\beta+\Delta+\bar{\beta}}\right)\right\},
$$

with the abbreviations

$$
\begin{aligned}
& \bar{\beta}=\frac{1}{s} \sqrt{s^{2}+\left(k_{+}^{2}\right)^{2}+\left(k_{-}^{2}\right)^{2}-2 s k_{+}^{2}-2 s k_{-}^{2}-2 k_{+}^{2} k_{-}^{2}}, \\
& \beta=\sqrt{1-\frac{4\left(M_{\mathrm{W}}^{2}-\mathrm{i} M_{\mathrm{W}} \Gamma_{\mathrm{W}}\right)}{s}}, \quad \Delta=\frac{\left|k_{+}^{2}-k_{-}^{2}\right|}{s} .
\end{aligned}
$$

\section{Treatment of soft and collinear photon emission}

We calculate the real photonic corrections from the full lowest-order matrix element of the process (2.4) without any expansion about the W-boson resonances. They are calculated from the integral

$$
\int \mathrm{d} \sigma^{\gamma \gamma \rightarrow 4 f \gamma}=\frac{1}{2 s} \int \mathrm{d} \Phi_{4 f \gamma}\left|\mathcal{M}^{\gamma \gamma \rightarrow 4 f \gamma}\right|^{2} \Theta\left(\Phi_{4 f \gamma}\right),
$$

where we have made the implementation of phase-space cuts explicit by including the step function $\Theta\left(\Phi_{4 f \gamma}\right)$, which is equal to 1 if an event passes the cuts and 0 otherwise. Since 
we evaluate the real matrix element $\mathcal{M}^{\gamma \gamma \rightarrow 4 f \gamma}$ with massless particles, the phase-space integral diverges in the soft and collinear regions, where the emitted photon is either soft or collinear to an outgoing external charged fermion. In these regions we reintroduce a formally infinitesimal photon mass $\lambda$ and small fermion masses $m_{f}$ as regulators.

To this end, we apply two different methods: the dipole subtraction and the (twocutoff) phase-space slicing methods. In the case of collinear-safe observables we closely follow the approach of Ref. [10] and only give a brief description in Section 4.1 since the procedure is very similar to the $\mathrm{e}^{+} \mathrm{e}^{-}$case. In Section 4.2 we describe how the two methods are extended to non-collinear-safe observables.

\subsection{Collinear-safe observables}

\subsubsection{Phase-space slicing}

In the phase-space slicing approach the phase space is divided into regions where the integrand is finite and can, thus, be integrated numerically, and regions where the integrand becomes singular. In the singular regions the integration over the photon phase space is carried out analytically in the approximation that the photon is soft and/or collinear to a charged fermion.

The singular regions consist of two parts one of which contains a soft photon $\left(k^{0}<\Delta E\right)$ and the other a photon that is collinear but not soft $\left(k^{0}>\Delta E\right.$ and $\theta_{\gamma f}<\Delta \theta$, where $\theta_{\gamma f}$ is the angle between the photon and a charged fermion). Thus, the real corrections are decomposed according to

$$
\int \mathrm{d} \sigma^{\gamma \gamma \rightarrow 4 f \gamma}=\int \mathrm{d} \sigma_{\text {soft }}+\int \mathrm{d} \sigma_{\text {coll }}+\int \mathrm{d} \sigma_{\text {finite }}^{\gamma \gamma \rightarrow 4 f \gamma}
$$

where the cutoff parameters $\Delta E$ and $\Delta \theta$ are defined in the CM system of the incoming photons. Both in the soft and collinear regions the squared matrix element $\left|\mathcal{M}^{\gamma \gamma \rightarrow 4 f \gamma}\right|^{2}$ factorizes into the squared lowest-order matrix element $\left|\mathcal{M}_{\mathrm{Born}}^{\gamma \gamma \rightarrow 4 f}\right|^{2}$ and a universal factor containing the singularity. The five-particle phase space also factorizes into a four-particle phase space and a photon part, so that $\mathrm{d} \sigma_{\text {soft }}$ and $\mathrm{d} \sigma_{\text {coll }}$ can be integrated over the photon momentum. Taking over the results from Ref. [10] yields

$$
\begin{aligned}
\mathrm{d} \sigma_{\text {soft }}=\mathrm{d} \sigma_{\text {Born }}^{\gamma \gamma \rightarrow 4 f} \Theta\left(\Phi_{4 f}\right) \frac{\alpha}{\pi} \sum_{i=1}^{4} \sum_{j=i+1}^{4}(-1)^{i+j} Q_{i} Q_{j}\left\{2 \ln \left(\frac{2 \Delta E}{\lambda}\right)\left[1-\ln \left(\frac{s_{i j}}{m_{i} m_{j}}\right)\right]\right. \\
\left.-\ln \left(\frac{4 p_{i}^{0} p_{j}^{0}}{m_{i} m_{j}}\right)+\ln ^{2}\left(\frac{2 p_{i}^{0}}{m_{i}}\right)+\ln ^{2}\left(\frac{2 p_{j}^{0}}{m_{j}}\right)+\frac{\pi^{2}}{3}+\operatorname{Li}_{2}\left(1-\frac{4 p_{i}^{0} p_{j}^{0}}{s_{i j}}\right)\right\}
\end{aligned}
$$

and

$$
\mathrm{d} \sigma_{\text {coll }}=\mathrm{d} \sigma_{\text {Born }}^{\gamma \gamma \rightarrow 4 f} \Theta\left(\Phi_{4 f}\right) \frac{\alpha}{2 \pi} \sum_{i=1}^{4} Q_{i}^{2}\left\{\left[\frac{3}{2}+2 \ln \left(\frac{\Delta E}{p_{i}^{0}}\right)\right]\left[1-2 \ln \left(\frac{\Delta \theta p_{i}^{0}}{m_{i}}\right)\right]+3-\frac{2 \pi^{2}}{3}\right\},
$$

where $Q_{i}$ and $m_{i}$ denote the relative electric charge and mass of fermion $f_{i}$, respectively. The step function $\Theta\left(\Phi_{4 f}\right)$ indicates that both $\mathrm{d} \sigma_{\text {soft }}$ and $\mathrm{d} \sigma_{\text {coll }}$ are defined on the fourparticle phase space of the lowest-order cross section, so that the singular part

$$
\mathrm{d} \sigma_{\text {sing }}^{\gamma \gamma \rightarrow 4 f \gamma}=\mathrm{d} \sigma_{\text {soft }}+\mathrm{d} \sigma_{\text {coll }}
$$


can be locally combined with the singular part of the virtual corrections, which are defined on the same phase space. In the result $\mathrm{d} \sigma_{\text {virt+real,sing }}^{\gamma \gamma \rightarrow 4 f}$ all dependences on the photon and fermion masses cancel.

While $\mathrm{d} \sigma_{\text {virt+real,sing }}^{\gamma \gamma \rightarrow 4 f}$ depends on the cutoff parameters $\Delta E$ and $\Delta \theta$ analytically, the finite real corrections $\int \mathrm{d} \sigma_{\text {finite }}^{\gamma \gamma \rightarrow 4 f \gamma}$ only show this dependence upon the cuts in the numerical integration. Nevertheless, the cutoff dependence has to cancel in the full result in the limit $\Delta E, \Delta \theta \rightarrow 0$. This is illustrated on the l.h.s. of Figures 3 and 4 where the relative correction factor $\delta=\sigma / \sigma_{\text {Born }}-1$ of the $4 f \operatorname{part}\left(\int \mathrm{d} \sigma_{\text {virt,finite,DPA }}^{\gamma \gamma \rightarrow 4 f}+\int \mathrm{d} \sigma_{\text {virt+real,sing }}^{\gamma \gamma \rightarrow 4 f}\right)$ and of the $4 f \gamma$ part $\int \mathrm{d} \sigma_{\text {finite }}^{\gamma \gamma \rightarrow 4 f \gamma}$ is shown as a function of the cutoff parameters $\Delta E$ and $\Delta \theta$. The cancellations of the cutoff dependence of the two contributions is shown on a smaller scale on the r.h.s. of Figures 3 and 4 . While terms of $\mathcal{O}\left(\Delta E / E_{\text {beam }}\right)$ and $\mathcal{O}(\Delta \theta)$ become visible for large values of the cutoff parameters, for smaller values a plateau is reached. The integration error increases with decreasing cutoff values, until for too small values the integration error is usually underestimated. As a result, we decided to take $\Delta E / E_{\text {beam }}=10^{-3}$ and $\Delta \theta=10^{-2}$ as default values.

\subsubsection{Dipole subtraction method}

In a subtraction method an auxiliary function is constructed that contains the same singularities as the real corrections. Subtracting this function from the real corrections, this difference can be integrated numerically. The next step is to perform the singular integration of the auxiliary function over the photon momentum analytically and to readd the result to the virtual corrections. In our case where soft and collinear singularities originate from final-state radiation only, the soft and collinear singularities completely cancel against their counterparts in the virtual corrections for collinear-safe observables.

In the dipole subtraction method $[22,23]$, which was originally proposed for QCD [32], the auxiliary function consists of different contributions labelled by all ordered combinations of two charged fermions $i$ and $j$, which are called emitter and spectator. These contributions contain the singularities connected with the emitter $i$. Since there are only charged particles in the final state in $\gamma \gamma \rightarrow 4 f$, the situation is simpler than for $\mathrm{e}^{+} \mathrm{e}^{-} \rightarrow 4 f$. Explicitly the auxiliary function, which is subtracted from the spin-summed squared bremsstrahlung matrix element, reads

$$
\begin{aligned}
\left|\mathcal{M}_{\mathrm{sub}}\right|^{2} & =\sum_{\substack{i, j=1 \\
i \neq j}}^{4}\left|\mathcal{M}_{\mathrm{sub}, i j}\right|^{2} \\
\left|\mathcal{M}_{\mathrm{sub}, i j}\left(\Phi_{4 f \gamma}\right)\right|^{2} & =-(-1)^{i+j} Q_{i} Q_{j} e^{2} g_{i j}^{(\mathrm{sub})}\left(p_{i}, p_{j}, k\right)\left|\mathcal{M}_{\mathrm{Born}}^{\gamma \gamma \rightarrow 4 f}\left(\tilde{\Phi}_{4 f, i j}\right)\right|^{2} .
\end{aligned}
$$

Adopting the formulation of Ref. $[22]^{4}$, the soft and collinear divergences are contained in the function

$$
g_{i j}^{(\mathrm{sub})}\left(p_{i}, p_{j}, k\right)=\frac{1}{\left(p_{i} k\right)\left(1-y_{i j}\right)}\left[\frac{2}{1-z_{i j}\left(1-y_{i j}\right)}-1-z_{i j}\right]
$$

\footnotetext{
${ }^{4}$ The formulation of Ref. [23] differs from that by the regular factor $1 /\left(1-y_{i j}\right)$ in Eq. (4.7), so that the readded singular contributions of Refs. [22] and [23] differ by non-singular finite parts.
} 

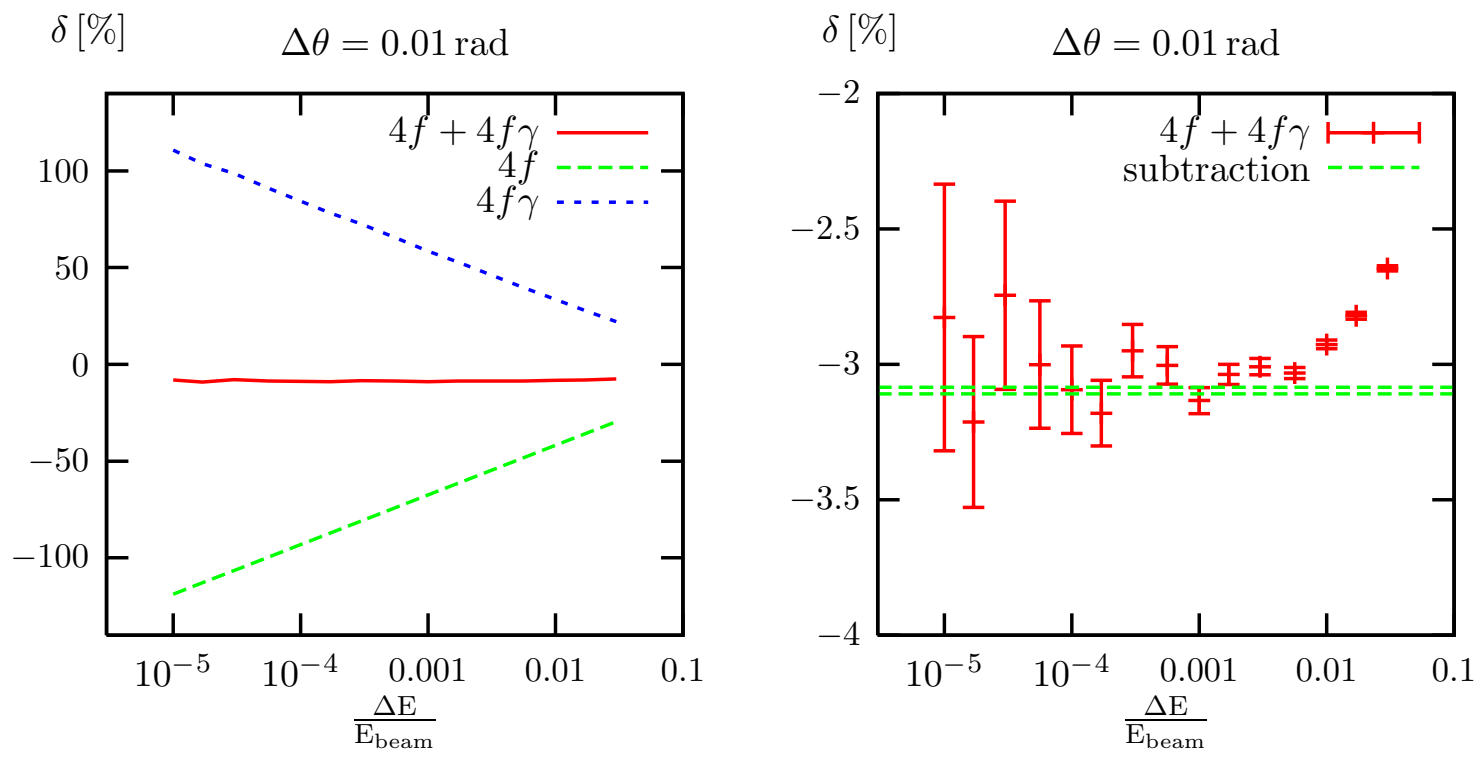

Figure 3: Dependence of the corrections on the energy cutoff in the slicing approach for the process $\gamma \gamma \rightarrow \nu_{\mathrm{e}} \mathrm{e}^{+} \mathrm{d} \overline{\mathrm{u}}$ at $\sqrt{s_{\gamma \gamma}}=500 \mathrm{GeV}$. For comparison the corresponding result obtained with the dipole subtraction method is shown as a $1 \sigma$ band in the plot on the r.h.s.
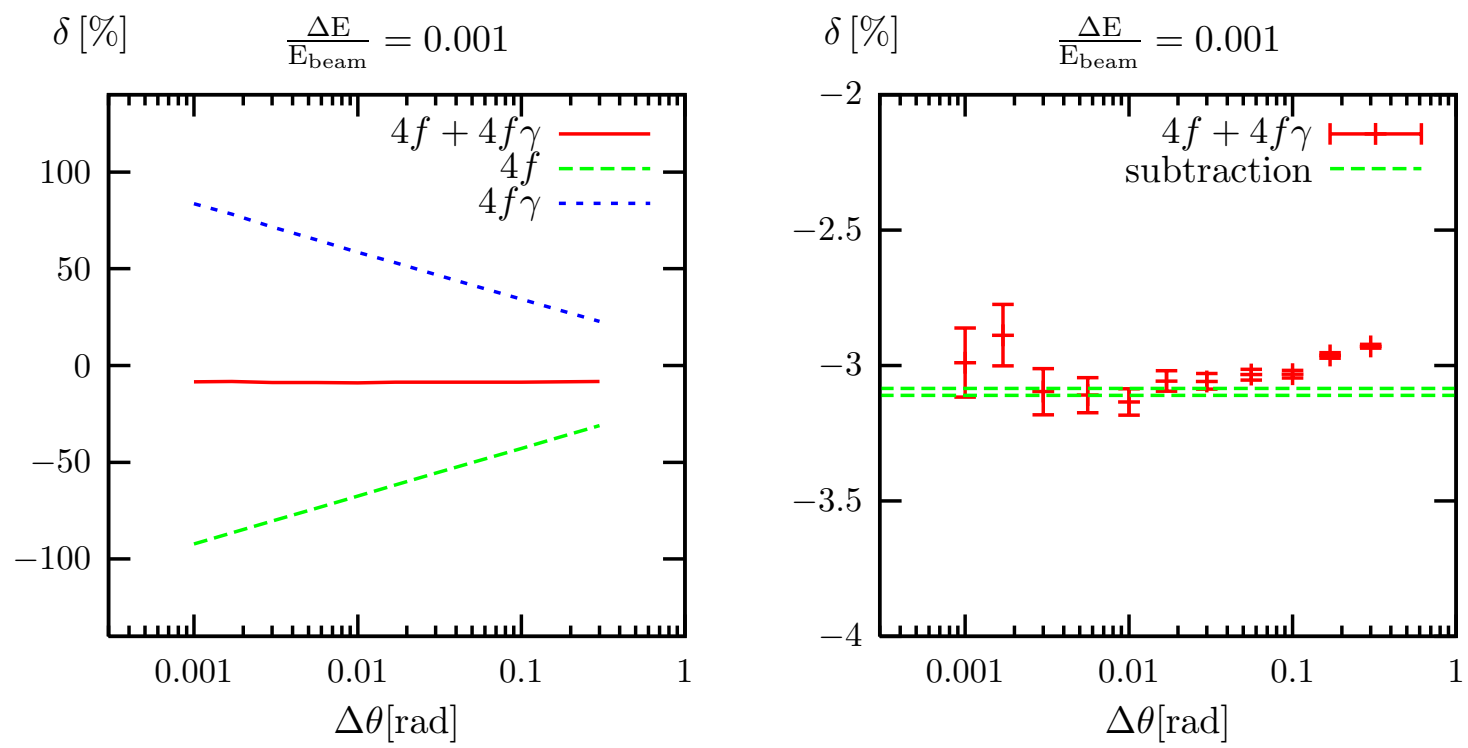

Figure 4: Dependence of the corrections on the angular cutoff in the slicing approach for the process $\gamma \gamma \rightarrow \nu_{\mathrm{e}} \mathrm{e}^{+} \mathrm{d} \overline{\mathrm{u}}$ at $\sqrt{s_{\gamma \gamma}}=500 \mathrm{GeV}$. For comparison the corresponding result obtained with the subtraction method is shown as a $1 \sigma$ band in the plot on the r.h.s. 
with

$$
y_{i j}=\frac{p_{i} k}{p_{i} p_{j}+p_{i} k+p_{j} k}, \quad z_{i j}=\frac{p_{i} p_{j}}{p_{i} p_{j}+p_{j} k} .
$$

The embedding of the $4 f$ phase space $\tilde{\Phi}_{4 f, i j}$ into the $4 f \gamma$ phase space $\Phi_{4 f \gamma}$ is defined as

$$
\tilde{p}_{i}^{\mu}=p_{i}^{\mu}+k^{\mu}-\frac{y_{i j}}{1-y_{i j}} p_{j}^{\mu}, \quad \tilde{p}_{j}^{\mu}=\frac{1}{1-y_{i j}} p_{j}^{\mu},
$$

with all other momenta unchanged, $\tilde{p}_{k}=p_{k}, k \neq i, j$. Subtracting the auxiliary function from the real corrections enables us to carry out the numerical integration,

$$
\int \mathrm{d} \sigma_{\text {finite }}^{\gamma \gamma \rightarrow 4 f \gamma}=\frac{1}{2 s} \int \mathrm{d} \Phi_{4 f \gamma}\left[\left|\mathcal{M}^{\gamma \gamma \rightarrow 4 f \gamma}\right|^{2} \Theta\left(\Phi_{4 f \gamma}\right)-\sum_{\substack{i, j=1 \\ i \neq j}}^{4}\left|\mathcal{M}_{\text {sub }, i j}\right|^{2} \Theta\left(\tilde{\Phi}_{4 f, i j}\right)\right],
$$

which does not contain any soft or collinear divergences by construction of $\left|\mathcal{M}_{\text {sub }}\right|^{2}$ for collinear-safe observables. In this context, it is important to notice the different arguments of the step functions $\Theta$ which account for phase-space cuts. Since for a generic point in $4 f \gamma$ phase space each $i j$ contribution corresponds to a different point in phase space, there is in general no correlation between the values of the different step functions. For collinearsafe observables, however, we have $\Theta\left(\Phi_{4 f \gamma}\right)=\Theta\left(\tilde{\Phi}_{4 f, i j}\right)$ in the soft region $(k \rightarrow 0)$ and in the region where the photon momentum $k$ is nearly collinear to the emitter momentum $p_{i}\left(p_{i} k \rightarrow 0\right)$. The collinear safety can, e.g., be enforced by photon recombination, as discussed in the next section in more detail.

In order to combine the subtraction function with the virtual correction, it has to be integrated over the photon momentum, yielding

$$
\int \mathrm{d} \sigma_{\text {sing }}^{\gamma \gamma \rightarrow 4 f \gamma}=-\frac{\alpha}{2 \pi} \sum_{\substack{i, j=1 \\ i \neq j}}^{4}(-1)^{i+j} Q_{i} Q_{j} \frac{1}{2 s} \int \mathrm{d} \Phi_{4 f} G_{i j}^{(\mathrm{sub})}\left(s_{i j}\right)\left|\mathcal{M}_{\mathrm{Born}}^{\gamma \gamma \rightarrow 4 f}\left(\Phi_{4 f}\right)\right|^{2} \Theta\left(\Phi_{4 f}\right) .
$$

The singularities are contained in the function

$$
G_{i j}^{(\mathrm{sub})}\left(s_{i j}\right)=\mathcal{L}\left(s_{i j}, m_{i}^{2}\right)-\frac{\pi^{2}}{3}+\frac{3}{2}
$$

with

$$
\mathcal{L}\left(s_{i j}, m_{i}^{2}\right)=\ln \left(\frac{m_{i}^{2}}{s_{i j}}\right) \ln \left(\frac{\lambda^{2}}{s_{i j}}\right)+\ln \left(\frac{\lambda^{2}}{s_{i j}}\right)-\frac{1}{2} \ln ^{2}\left(\frac{m_{i}^{2}}{s_{i j}}\right)+\frac{1}{2} \ln \left(\frac{m_{i}^{2}}{s_{i j}}\right) .
$$

We have numerically checked that these soft and collinear divergences are completely cancelled by their counterparts in the virtual correction.

\subsection{Non-collinear-safe observables}

In the previous sections the matching of real and virtual corrections was described for collinear-safe observables. We speak of collinear-safe observables if a nearly collinear system of a charged fermion and a photon is treated inclusively, i.e. if phase-space selection 
cuts (or histogram bins of distributions) depend only on the sum $p_{i}+k$ of the nearly collinear fermion and photon momenta. In this case the energy fraction

$$
z_{i}=\frac{p_{i}^{0}}{p_{i}^{0}+k^{0}}
$$

of a charged fermion $f_{i}$ after emitting a photon in a sufficiently small cone around its direction of flight is fully integrated over, because it is not constrained by any phase-space cut (or histogram bin selection in distributions). Thus, the KLN theorem [33] guarantees that all singularities connected with final-state radiation cancel between the virtual and real corrections, even though they are defined on different phase spaces. A sufficient inclusiveness is, e.g., achieved by the photon recombination described in Section 5.1, which treats outgoing charged fermions and photons as one quasi-particle if they are very close in angle.

In the previous section we could, therefore, integrate the subtraction function $\left|\mathcal{M}_{\text {sub }}\right|^{2}$ and the slicing contribution $\mathrm{d} \sigma_{\text {coll }}$ over $z_{i}$ analytically. In this section we are concerned with non-collinear-safe observables, i.e. the fermion-photon system is not treated inclusively and fermion-mass singularities can become visible. As the integration over $z_{i}$ now is constrained by phase-space cuts (or histogram bins), we have to modify the methods described in the previous section in such a way that the integration over $z_{i}$ is part of the numerical phase-space integration.

\subsubsection{Phase-space slicing}

In the slicing method the procedure is straightforward. The numerical integration over $z=z_{i}$ in the collinear parts reads

$$
\begin{gathered}
\mathrm{d} \sigma_{\text {coll }}=\mathrm{d} \sigma_{\text {Born }}^{\gamma \gamma \rightarrow 4 f}\left(\tilde{\Phi}_{4 f}\right) \frac{\alpha}{2 \pi} \sum_{i=1}^{4} Q_{i}^{2} \int_{0}^{1-\Delta E / \tilde{p}_{i}^{0}} \mathrm{~d} z \Theta\left(p_{i}=z \tilde{p}_{i}, k=(1-z) \tilde{p}_{i},\left\{\tilde{p}_{j \neq i}\right\}\right) \\
\times\left\{P_{f f}(z)\left[2 \ln \left(\frac{\Delta \theta \tilde{p}_{i}^{0}}{m_{i}} z\right)-1\right]+(1-z)\right\}
\end{gathered}
$$

with the splitting function

$$
P_{f f}(z)=\frac{1+z^{2}}{1-z}
$$

The Born cross section and the logarithm still depend on the momenta of the $4 f$ phase space $\tilde{\Phi}_{4 f}$ which are labelled $\tilde{p}_{i}$. In the cut and recombination function $\Theta$, however, the momentum $\tilde{p}_{i}$ of the fermion $i$ (before photon emission) is distributed to the fermion momentum $p_{i}$ and the photon momentum $k$. For collinear-safe observables, as e.g. achieved by photon recombination, the $\Theta$ function effectively only depends on the sum $p_{i}+k=\tilde{p}_{i}$ of the collinear momenta, which is independent of $z$. In this case, the $\Theta$ function becomes

$\Theta\left(\tilde{\Phi}_{4 f}\right)$, and the $z$-integration can be easily carried out analytically yielding Eq. (4.4).

\subsubsection{Dipole subtraction method}

In the case of the dipole subtraction method the generalization to non-collinear-safe observables is more complicated than in the slicing approach, since the integration over 
the photon momentum is more involved. Here, we collect the formulas relevant for our calculation. Details on their derivation are given in App. B.

In order to keep the information on the energy fraction $z$ in each part of the subtraction function, the finite part of the real corrections is modified to

$$
\begin{aligned}
\int \mathrm{d} \sigma_{\text {finite }}^{\gamma \gamma \rightarrow 4 f \gamma}= & \frac{1}{2 s} \int \mathrm{d} \Phi_{4 f \gamma}\left[\left|\mathcal{M}^{\gamma \gamma \rightarrow 4 f \gamma}\right|^{2} \Theta\left(\Phi_{4 f \gamma}\right)\right. \\
& \left.-\sum_{\substack{i, j=1 \\
i \neq j}}^{4}\left|\mathcal{M}_{\text {sub }, i j}\right|^{2} \Theta\left(p_{i}=z_{i j} \tilde{p}_{i}, k=\left(1-z_{i j}\right) \tilde{p}_{i},\left\{\tilde{p}_{k \neq i}\right\}\right)\right] .
\end{aligned}
$$

It is easily seen that the variable $z_{i j}$, which is defined in Eq. (4.8), plays the role of the energy fraction $z_{i}$ in the collinear limit for each dipole $i j$. Again, in the collinear-safe case the $\Theta$ functions of the subtraction function depend only on the sums $p_{i}+k=\tilde{p}_{i}$ of collinear momenta; in this case we recover Eq. (4.10).

In the integration of the subtraction function over the photon phase space, we now have to leave the integrations over $z_{i j}$ open. The resulting $z_{i j}$ dependence of the integrand is most conveniently described with a $[\ldots]_{+}$prescription $^{5}$, which separates the soft singularity at $z_{i j}=1$. The endpoint part at $z_{i j}=1$, which results from the full integration over $z_{i j}$, exactly corresponds to the contribution of $G_{i j}^{(\mathrm{sub})}\left(\tilde{s}_{i j}\right)$ for the collinear-safe case, as given in Eq. (4.12), where $\tilde{s}_{i j}=2 \tilde{p}_{i} \tilde{p}_{j}$. The continuum part in $z_{i j}$ involves an integral over $\left[\overline{\mathcal{G}}_{i j}^{(\mathrm{sub})}\left(\tilde{s}_{i j}, z_{i j}\right)\right]_{+}$with

$$
\overline{\mathcal{G}}_{i j}^{(\mathrm{sub})}\left(\tilde{s}_{i j}, z\right)=P_{f f}(z)\left[\ln \left(\frac{\tilde{s}_{i j} z}{m_{i}^{2}}\right)-1\right]+(1+z) \ln (1-z)+(1-z) .
$$

The total integrated subtraction part explicitly reads

$$
\begin{aligned}
\int \mathrm{d} \sigma_{\text {sing }}^{\gamma \gamma \rightarrow 4 f \gamma}= & -\frac{\alpha}{2 \pi} \sum_{\substack{i, j=1 \\
i \neq j}}^{4}(-1)^{i+j} Q_{i} Q_{j} \frac{1}{2 s} \int \mathrm{d} \tilde{\Phi}_{4 f, i j}\left|\mathcal{M}_{\text {Born }}^{\gamma \gamma \rightarrow 4 f}\left(\tilde{\Phi}_{4 f, i j}\right)\right|^{2}\left\{G_{i j}^{(\mathrm{sub})}\left(\tilde{s}_{i j}\right) \Theta\left(\tilde{\Phi}_{4 f, i j}\right)\right. \\
& \left.+\int_{0}^{1} \mathrm{~d} z\left[\overline{\mathcal{G}}_{i j}^{(\text {sub })}\left(\tilde{s}_{i j}, z\right)\right]_{+} \Theta\left(p_{i}=z \tilde{p}_{i}, k=(1-z) \tilde{p}_{i},\left\{\tilde{p}_{k \neq i}\right\}\right)\right\} .
\end{aligned}
$$

Owing to the $[\ldots]_{+}$prescription, the continuum part is zero if the full integration over $z$ is carried out, thereby recovering the collinear-safe case (4.11).

\footnotetext{
${ }^{5}$ We use the definition $\int_{0}^{1} \mathrm{~d} x[f(x)]_{+} g(x) \equiv \int_{0}^{1} \mathrm{~d} x f(x)[g(x)-g(1)]$.
} 


\section{$5 \quad$ Numerical results}

\section{$5.1 \quad$ Input parameters and setup}

We use the following set of input parameters [34]:

$$
\begin{aligned}
& G_{\mu}=1.16639 \times 10^{-5} \mathrm{GeV}^{-2}, \quad \alpha(0)=1 / 137.03599976, \quad \alpha_{\mathrm{s}}=0.1172, \\
& M_{\mathrm{W}}=80.423 \mathrm{GeV}, \quad \Gamma_{\mathrm{W}}=2.118 \mathrm{GeV}, \\
& M_{\mathrm{Z}}=91.1876 \mathrm{GeV}, \quad \Gamma_{\mathrm{Z}}=2.4952 \mathrm{GeV}, \\
& m_{\mathrm{e}}=0.510998902 \times 10^{-3} \mathrm{GeV}, \quad m_{\mu}=0.105658357 \mathrm{GeV}, \quad m_{\tau}=1.77699 \mathrm{GeV} \text {, } \\
& m_{\mathrm{u}}=0.066 \mathrm{GeV}, \quad m_{\mathrm{c}}=1.2 \mathrm{GeV}, \quad m_{\mathrm{t}}=174.3 \mathrm{GeV}, \\
& m_{\mathrm{d}}=0.066 \mathrm{GeV}, \quad m_{\mathrm{s}}=0.15 \mathrm{GeV}, \quad m_{\mathrm{b}}=4.3 \mathrm{GeV} .
\end{aligned}
$$

If not stated otherwise, the Higgs mass is $M_{\mathrm{H}}=170 \mathrm{GeV}$. In some cases we alternatively use $M_{\mathrm{H}}=130 \mathrm{GeV}$. The corresponding values for the Higgs-boson decay width $\Gamma_{\mathrm{H}}$, which have been obtained with the program HDECAY [35], are given by

$$
\Gamma_{\mathrm{H}}\left(M_{\mathrm{H}}=170 \mathrm{GeV}\right)=0.3834 \mathrm{GeV}, \quad \Gamma_{\mathrm{H}}\left(M_{\mathrm{H}}=130 \mathrm{GeV}\right)=0.004995 \mathrm{GeV} .
$$

We set the quark-mixing matrix to the unit matrix throughout, but in the limit of massless external fermions a non-trivial quark-mixing matrix can be included by a simple rescaling of the cross sections.

Furthermore, we apply a set of recombination and separation cuts:

1. Bremsstrahlung photons that are closer than $5^{\circ}$ to a charged fermion or have less energy than $1 \mathrm{GeV}$ are recombined with the charged fermion that is closest in angle. This means that in this case before evaluating distributions or applying phase-space cuts the momenta of the photon and the fermion are added and associated with the fermion, while the photon is discarded.

2. The following separation cuts are applied to the momenta defined after a possible recombination:

$$
\begin{aligned}
E_{l} & >10 \mathrm{GeV}, & \theta(l, \text { beam }) & >5^{\circ}, \\
E_{q} & >10 \mathrm{GeV}, & \theta\left(l, l^{\prime}\right) & >5^{\circ}, \quad \theta(l, q)>5^{\circ},
\end{aligned}
$$

where an obvious notation for energies $E_{\ldots}$, angles $\theta(\ldots)$, and invariant masses $m(\ldots)$ for leptons $l$ and quarks $q$ is used.

The separation cuts and input parameters are the same as in Ref. $[3]^{6}$ for the processes $\gamma \gamma \rightarrow 4 f$, so that we reproduce the Born cross sections that we calculated there. In particular, we exclude forward and backward scattered charged fermions, because they cause collinear singularities. While for final-state quarks these singularities signal a nonperturbative regime, for leptons they are in principle cured by finite-mass effects. However, we exclude this region by demanding that leptons appear in the detector with finite

\footnotetext{
${ }^{6}$ There is a misprint in Eq. (6.1) of Ref. [3]. The value for $\alpha_{s}$ is supposed to be $\alpha_{s}=0.1172$ and not $\alpha_{s}=1.1172$.
} 
production angle and energy. Compared to Ref. [10] we use different recombination cuts, because, in contrast to $\mathrm{e}^{+} \mathrm{e}^{-}$collisions, the recombination criterion based on invariant masses does not lead to collinear-safe observables. This is due to the collinear singularity that arises if a charged fermion is collinear to the beam. Even though an appropriate cut on the angle between charged fermions and the beam is imposed, it might happen that a photon with relatively high energy is recombined with a low-energy fermion that is close to the beam. Thus, after recombination, the fermion almost follows the direction of the photon and is not affected by the angular cut. Such events are avoided by taking a recombination condition based on the angle.

For the evaluation of the lowest-order matrix elements of $\gamma \gamma \rightarrow 4 f$ and $\gamma \gamma \rightarrow 4 f \gamma$, we use the fixed-width scheme, in which the gauge-boson width is introduced in all (i.e. time- and space-like) propagators. As argued in Section 2.4 of Ref. [3], this scheme does not break gauge invariance for reactions $\gamma \gamma \rightarrow 4 f(+\gamma)$ with massless external fermions.

The photon spectrum is accounted for by using the parametrization of the program CompAZ [4], as described in Section 5 of Ref. [3]. In order to distinguish the cases with

and without convolution over the photon spectrum, we write $\sqrt{s_{\mathrm{ee}}}$ and $\sqrt{s_{\gamma \gamma}}$ for the CM energies in these cases, respectively.

In the numerical integration we generate $2 \cdot 10^{7}$ events for the plots showing the integrated cross sections, and $5 \cdot 10^{7}$ events for distributions and for the integrated cross sections in Table 1. If not stated otherwise, the shown results are based on the subtraction method, but have been cross-checked with the slicing approach. Moreover, we have additionally checked most of the results by reproducing them within statistical uncertainties with our second independent Monte Carlo generator.

\subsection{Integrated cross sections}

In Table 1 we present a survey of integrated cross sections for a leptonic, a hadronic, and two semi-leptonic final states, as obtained with the subtraction and slicing methods. The cross sections of the semi-leptonic final states differ because of the effective polarizations of the photons resulting from the Compton backscattering (cf. Section 6.3 of Ref. [3]). Final states that differ only in the fermion generation (i.e. in their mass values) receive the same radiative corrections, since our predictions are based on the massless limit for the external fermions and mass singularities cancel after performing a photon recombination. The results obtained with the two methods for treating the real corrections, subtraction ("sub") and slicing ("sli"), are in good agreement. Note that they both are implemented in the same Monte Carlo generator, which, thus, yields identical results for $\sqrt{s_{\gamma \gamma}}<170 \mathrm{GeV}$ where the IBA is used. This is the reason why the "sub" and "sli" numbers are identical in the case of $\sqrt{s_{\mathrm{ee}}}=200 \mathrm{GeV}$ with $\gamma$ spectrum, where only the range $\sqrt{s_{\gamma \gamma}}<170 \mathrm{GeV}$ is relevant in the convolution.

In Figure 5 the integrated cross section for $\gamma \gamma \rightarrow \nu_{\mathrm{e}} \mathrm{e}^{+} \mathrm{d} \overline{\mathrm{u}}$ including radiative corrections is compared with the Born cross section as a function of the CM energy for monochromatic photon beams. The "best" curves correspond to the $\mathcal{O}(\alpha)$-corrected cross sections. A Higgs boson of $M_{\mathrm{H}}=170 \mathrm{GeV}$ produces a sharp peak in the cross section at $\sqrt{s_{\gamma \gamma}}=$ $170 \mathrm{GeV}$, while for larger energies the corrections are almost independent of the Higgs mass. The relative corrections $\delta=\sigma / \sigma_{\text {Born }}-1$ in the four lower plots of Figure 5 behave roughly like the corrections to on-shell W-pair production [15-17]. Close to the W-pair 


\begin{tabular}{|c|c|c|c|c|c|}
\hline & & \multicolumn{2}{|c|}{$\sigma[\mathrm{fb}]$} & \multirow[t]{2}{*}{$\sigma_{\text {Born }}[\mathrm{fb}]$} & \\
\hline CM energy & final state & subtraction & slicing & & $($ sub-sli)/sli \\
\hline \multirow{4}{*}{$\begin{array}{c}\sqrt{s_{\gamma \gamma}}=200 \mathrm{GeV} \\
\text { without } \gamma \text { spectrum }\end{array}$} & $\nu_{\mathrm{e}} \mathrm{e}^{+} \mu^{-} \bar{\nu}_{\mu}$ & $581.403(67)$ & $581.41(16)$ & $575.628(64)$ & $0.00(3) \%$ \\
\hline & $\nu_{\mathrm{e}} \mathrm{e}^{+} \mathrm{d} \overline{\mathrm{u}}$ & $1734.02(23)$ & $1735.26(43)$ & $1716.10(22)$ & $-0.07(3) \%$ \\
\hline & $\mathrm{ud} \mathrm{e}^{-} \bar{\nu}_{\mathrm{e}}$ & $1734.24(23)$ & $1734.32(43)$ & $1716.06(22)$ & $0.00(3) \%$ \\
\hline & $\mathrm{u} \overline{\mathrm{d}} \mathrm{s} \overline{\mathrm{c}}$ & $4931.01(76)$ & $4935.0(1.0)$ & $4878.67(73)$ & $-0.08(3) \%$ \\
\hline \multirow{4}{*}{$\begin{array}{c}\sqrt{s_{\gamma \gamma}}=500 \mathrm{GeV} \\
\text { without } \gamma \text { spectrum }\end{array}$} & $\nu_{\mathrm{e}} \mathrm{e}^{+} \mu^{-} \bar{\nu}_{\mu}$ & $801.21(11)$ & $801.57(20)$ & $826.620(91)$ & $-0.05(3) \%$ \\
\hline & $\nu_{\mathrm{e}} \mathrm{e}^{+} \mathrm{d} \overline{\mathrm{u}}$ & $2278.50(34)$ & $2279.96(51)$ & $2351.37(30)$ & $-0.06(3) \%$ \\
\hline & $\mathrm{ud \overline {d }} \mathrm{e}^{-} \bar{\nu}_{\mathrm{e}}$ & $2278.45(34)$ & $2278.84(48)$ & $2351.39(30)$ & $-0.02(3) \%$ \\
\hline & $\mathrm{u} \overline{\mathrm{d}} \mathrm{s} \overline{\mathrm{c}}$ & $6452.2(1.0)$ & $6452.8(1.2)$ & $6662.25(96)$ & $-0.01(2) \%$ \\
\hline \multirow{4}{*}{$\begin{array}{c}\sqrt{s_{\gamma \gamma}}=1000 \mathrm{GeV} \\
\text { without } \gamma \text { spectrum }\end{array}$} & $\nu_{\mathrm{e}} \mathrm{e}^{+} \mu^{-} \bar{\nu}_{\mu}$ & $696.25(15)$ & $696.68(17)$ & $746.995(93)$ & $-0.06(3) \%$ \\
\hline & $\nu_{\mathrm{e}} \mathrm{e}^{+} \mathrm{d} \overline{\mathrm{u}}$ & $1836.31(43)$ & $1836.96(45)$ & $1979.92(29)$ & $-0.04(3) \%$ \\
\hline & $\mathrm{ud \overline {de }}^{-} \bar{\nu}_{\mathrm{e}}$ & $1836.37(42)$ & $1836.95(42)$ & $1979.95(29)$ & $-0.03(3) \%$ \\
\hline & $\mathrm{u} \overline{\mathrm{d}} \mathrm{s} \overline{\mathrm{C}}$ & $4892.2(1.2)$ & $4891.4(1.1)$ & $5300.97(90)$ & $0.02(3) \%$ \\
\hline \multirow{4}{*}{$\begin{array}{l}\sqrt{s_{\mathrm{ee}}}=200 \mathrm{GeV} \\
\text { with } \gamma \text { spectrum }\end{array}$} & $\nu_{\mathrm{e}} \mathrm{e}^{+} \mu^{-} \bar{\nu}_{\mu}$ & $0.073205(44)$ & $0.073205(44)$ & $0.072009(44)$ & 0 \\
\hline & $\nu_{\mathrm{e}} \mathrm{e}^{+} \mathrm{d} \overline{\mathrm{u}}$ & $0.33129(21)$ & $0.33129(21)$ & $0.32601(21)$ & 0 \\
\hline & $\mathrm{ud \overline {d }} \mathrm{e}^{-} \bar{\nu}_{\mathrm{e}}$ & $0.39204(25)$ & $0.39204(25)$ & $0.38593(24)$ & 0 \\
\hline & $\mathrm{u} \overline{\mathrm{d}} \mathrm{s} \overline{\mathrm{c}}$ & $1.24460(79)$ & $1.24460(79)$ & $1.22537(78)$ & 0 \\
\hline \multirow{4}{*}{$\begin{array}{l}\sqrt{s_{\mathrm{ee}}}=500 \mathrm{GeV} \\
\text { with } \gamma \text { spectrum }\end{array}$} & $\nu_{\mathrm{e}} \mathrm{e}^{+} \mu^{-} \bar{\nu}_{\mu}$ & $190.757(60)$ & $190.835(96)$ & $190.816(45)$ & $-0.04(6) \%$ \\
\hline & $\nu_{\mathrm{e}} \mathrm{e}^{+} \mathrm{d} \overline{\mathrm{u}}$ & $559.18(18)$ & $559.63(24)$ & $558.50(14)$ & $-0.08(5) \%$ \\
\hline & $\mathrm{ude}^{-} \bar{\nu}_{\mathrm{e}}$ & $564.58(18)$ & $564.79(25)$ & $565.05(14)$ & $-0.04(5) \%$ \\
\hline & $\mathrm{u} \overline{\mathrm{d}} \mathrm{s} \overline{\mathrm{c}}$ & $1604.92(54)$ & $1605.60(59)$ & $1603.80(45)$ & $-0.04(5) \%$ \\
\hline \multirow{4}{*}{$\begin{array}{l}\sqrt{s_{\mathrm{ee}}}=1000 \mathrm{GeV} \\
\text { with } \gamma \text { spectrum }\end{array}$} & $\nu_{\mathrm{e}} \mathrm{e}^{+} \mu^{-} \bar{\nu}_{\mu}$ & $165.759(91)$ & $165.604(81)$ & $170.588(41)$ & $0.09(7) \%$ \\
\hline & $\nu_{\mathrm{e}} \mathrm{e}^{+} \mathrm{d} \overline{\mathrm{u}}$ & $461.02(20)$ & 461.34(23) & $474.81(12)$ & $-0.07(7) \%$ \\
\hline & $\mathrm{u}_{\overline{\mathrm{d}}} \mathrm{e}^{-} \bar{\nu}_{\mathrm{e}}$ & $472.10(19)$ & $471.61(24)$ & $485.65(13)$ & $0.10(7) \%$ \\
\hline & $\mathrm{u} \overline{\mathrm{d}} \mathrm{s} \overline{\mathrm{c}}$ & $1296.49(52)$ & $1295.29(62)$ & $1335.13(38)$ & $0.09(6) \%$ \\
\hline
\end{tabular}

Table 1: Integrated cross sections for different final states and energies with and without convolution over the photon spectrum. The third column shows the result obtained with the subtraction method and the fourth with the slicing method. The last two columns show the Born cross section and the relative difference between subtraction and slicing. 

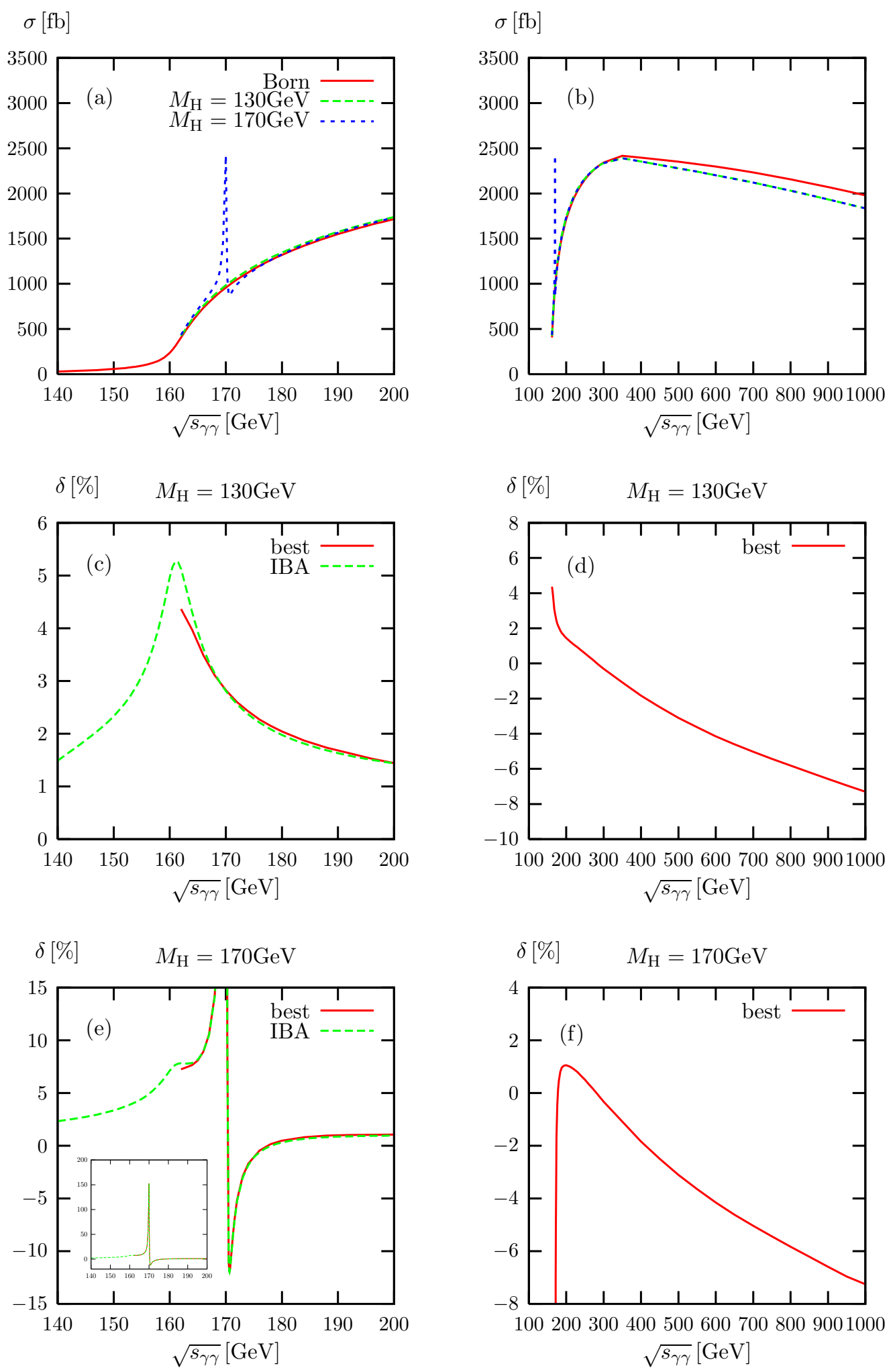

Figure 5: Integrated cross section for $\gamma \gamma \rightarrow \nu_{\mathrm{e}} \mathrm{e}^{+} \mathrm{d} \overline{\mathrm{u}}$ (the two upper plots) and relative radiative corrections (the four lower plots) without convolution over the photon spectrum for Higgs masses $M_{\mathrm{H}}=130 \mathrm{GeV}$ and $170 \mathrm{GeV}$. 


\begin{tabular}{|c|c|c|c|c|c|c|c|}
\hline$\sqrt{s_{\mathrm{ee}}} / \mathrm{GeV}$ & 200 & 240 & 260 & 280 & 300 & 500 & 1000 \\
\hline $\mathrm{TU}$ & $2.0 \%$ & $1.9 \%$ & $1.3 \%$ & $0.8 \%$ & $0.7 \%$ & $0.5 \%$ & $0.5 \%$ \\
\hline
\end{tabular}

Table 2: Estimates of the TU (5.4) for the $\mathcal{O}(\alpha)$-corrected cross section of $\gamma \gamma \rightarrow \nu_{\mathrm{e}} \mathrm{e}^{+} \mathrm{du}$ at various CM energies $\sqrt{s_{\mathrm{ee}}}$.

production threshold the corrections are dominated by the Coulomb singularity. For higher energies the corrections decrease until they reach about $-7 \%$ at $1 \mathrm{TeV}$. In this region they are dominated by large logarithms from the Regge and Sudakov domains.

In Figure 5(c) we also show the comparison with the IBA for a Higgs mass of $M_{\mathrm{H}}=$ $130 \mathrm{GeV}$. Since close to the W-pair production threshold the bulk of the corrections is due to the Coulomb singularity and since there are no other pronounced corrections, the agreement between the two curves is quite good. The very good agreement of the DPA and the IBA at $\sqrt{s_{\gamma \gamma}} \sim 170 \mathrm{GeV}$ both for semi-leptonic and for hadronic final states (in both cases the difference is well below $0.1 \%$ ) is of course accidental. For the leptonic final state the difference is about $0.7 \%$.

As explained in Section 3.5, the intrinsic uncertainty of the IBA is about $\sim \pm 2 \%$, while the DPA accuracy is up to $\lesssim 0.5 \%$ where it is applicable. Since the convolution of the hard $\gamma \gamma$ cross section, in general, involves both the IBA (in the low-energy tail) and the DPA (for $\sqrt{s_{\gamma \gamma}}>170 \mathrm{GeV}$ ), the uncertainty of our cross-section prediction is in the range $0.5-2 \%$, depending on the contribution of the IBA part to the full convolution. Denoting the IBA and DPA parts of the full cross section as $\Delta \sigma_{\mathrm{IBA}}$ and $\Delta \sigma_{\mathrm{DPA}}$ (both including the corresponding lowest-order contribution, so that $\left.\Delta \sigma_{\mathrm{IBA}}+\Delta \sigma_{\mathrm{DPA}}=\sigma\right)$, we can estimate the theoretical uncertainty (TU) of the corrected cross section $\sigma$ to

$$
\mathrm{TU}=\frac{\Delta \sigma_{\mathrm{IBA}}}{\sigma} \times 2 \%+\frac{\Delta \sigma_{\mathrm{DPA}}}{\sigma} \times 0.5 \% \text {. }
$$

Table 5.2 illustrates this estimate for a few CM energies $\sqrt{s_{\mathrm{ee}}}$ for $\gamma \gamma \rightarrow \nu_{\mathrm{e}} \mathrm{e}^{+} \mathrm{d} \overline{\mathrm{u}}$. For $\sqrt{s_{\mathrm{ee}}} \lesssim 230 \mathrm{GeV}$ our prediction possesses a TU of $\sim 2 \%$, because it is mainly based on the IBA, but already for $\sqrt{s_{\text {ee }}} \gtrsim 300 \mathrm{GeV}(500 \mathrm{GeV})$ the IBA contribution is widely suppressed so that the DPA uncertainty of $\lesssim 0.7 \%(0.5 \%)$ sets the precision of our calculation. We note, however, that the overall uncertainty of our calculation certainly becomes worse as soon as $\mathrm{TeV}$ energies for $\sqrt{s_{\gamma \gamma}}$ are dominating because of the relevance of high-energy logarithms beyond $\mathcal{O}(\alpha)$.

In Figure 5(e) the comparison of the full correction with the IBA is shown for a Higgs mass of $M_{\mathrm{H}}=170 \mathrm{GeV}$. The IBA includes the Higgs resonance via an effective coupling and reflects the shape of the resonance quite well.

The cross section including the convolution over the photon spectrum as a function of CM energy is shown in Figure 6 for a Higgs mass of $M_{\mathrm{H}}=130 \mathrm{GeV}$ and in the lower left plot also for $M_{\mathrm{H}}=170 \mathrm{GeV}$. In the upper plots the integrated cross sections are shown, and in the lower plots the corrections relative to the Born cross section. Recall that we use the IBA below $\sqrt{s_{\gamma \gamma}}=170 \mathrm{GeV}$. This means, in particular, that the Higgs resonance is calculated from the effective coupling and not from the full DPA in this region. The interesting structure in the lower left plot reflects the shape of the photon spectrum convoluted with the Higgs resonance. Since the Higgs resonance is very narrow, 

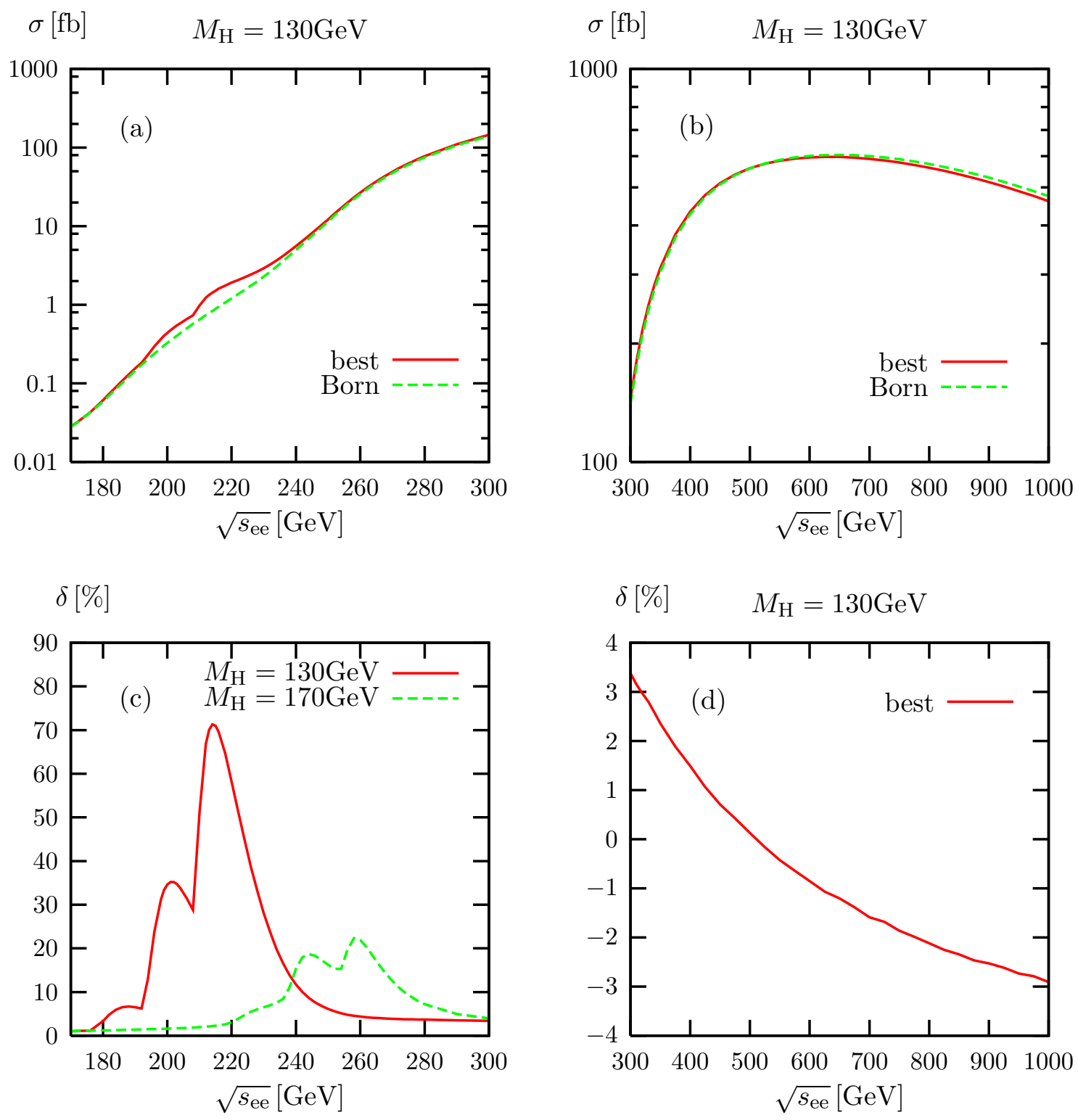

Figure 6: Integrated cross section for $\gamma \gamma \rightarrow \nu_{\mathrm{e}} \mathrm{e}^{+} \mathrm{d} \overline{\mathrm{u}}$ (upper plots) and relative radiative corrections (lower plots) including the convolution over the photon spectrum for Higgs masses of $M_{\mathrm{H}}=130 \mathrm{GeV}$ and $170 \mathrm{GeV}$ (lower left plot). For $\sqrt{s_{\mathrm{ee}}}>300 \mathrm{GeV}$ (shown on the r.h.s.) the "best" curve for $M_{\mathrm{H}}=170 \mathrm{GeV}$ practically coincides with the shown curve for $M_{\mathrm{H}}=130 \mathrm{GeV}$. 
a sizable contribution is only possible if $x_{1} x_{2} s_{\mathrm{ee}} \approx M_{\mathrm{H}}^{2}$ where $x_{1}$ and $x_{2}$ are the energy fractions carried by the photons. The correction is very small at low $\sqrt{s_{\mathrm{ee}}}$ where $x_{1}$ and $x_{2}$ have to be so large in order to match this condition that the corresponding spectrum is extremely small. Increasing $\sqrt{s_{\mathrm{ee}}}$ allows for lower values of $x_{1}$ and $x_{2}$. For instance, for $M_{\mathrm{H}}=130 \mathrm{GeV}$, the rise at $\sqrt{s_{\mathrm{ee}}} \sim 180 \mathrm{GeV}$ results from a region where both $x_{1}$ and $x_{2}$ are in the high-energy tail of the spectrum which is produced by multiple photon scattering. The peak at $\sqrt{s_{\text {ee }}} \sim 200 \mathrm{GeV}$ is caused by events where one photon comes from the high-energy tail and one from the dominant peak in the photon spectrum. Finally,

at $\sqrt{s_{\text {ee }}} \gtrsim 210 \mathrm{GeV}$ both $x_{1}$ and $x_{2}$ originate from the dominant photon-spectrum peak which causes the steep rise until $\sqrt{s_{\text {ee }}} \sim 220 \mathrm{GeV}$.

\subsection{Distributions}

In Figure 7 we show the invariant-mass distributions for the $\nu_{\mathrm{e}} \mathrm{e}^{+}$and $\mathrm{d} \overline{\mathrm{u}}$ pairs in the process $\gamma \gamma \rightarrow \nu_{\mathrm{e}} \mathrm{e}^{+} \mathrm{d} \overline{\mathrm{u}}$, both with and without convolution over the photon spectrum. The upper plots show the absolute predictions, and the lower plots the corrections normalized to the Born predictions. Since we use $\sqrt{s_{\gamma \gamma}}=500 \mathrm{GeV}$ or $\sqrt{s_{\mathrm{ee}}}=500 \mathrm{GeV}$, the corrections are shifted upwards when including the photon spectrum, because the effective energy of the photons is lower (cf. Figure 5). The shape of the corrections, however, is hardly changed by the convolution over the photon spectrum. As the shape of the corrections determine a possible shift of the peak of the invariant-mass distribution, it is of particular importance in the determination of the W-boson mass. The measurement of the W-boson mass can, e.g., be used for understanding and calibrating the detector of a $\gamma \gamma$ collider.

The distribution in the W-boson production angle is sensitive to anomalous couplings. In order to set bounds on these couplings it is mandatory to know radiative corrections, because both anomalous couplings and radiative corrections typically distort angular distributions. The corresponding angular distribution of the dū system, which is equal within the statistical error to the distribution of the $\nu_{\mathrm{e}} \mathrm{e}^{+}$system, is shown in Figure 8. While the correction without the photon spectrum is about $-9 \%$ for $\mathrm{W}$ bosons emitted perpendicular to the beam, the corrections are rather small when including the photon spectrum. As already explained above, the cross section is dominated by a region where the $\gamma \gamma \mathrm{CM}$ energy is smaller. In fact, the relative correction $\delta$ is accidentally small at $\sqrt{s_{\text {ee }}} \sim 500 \mathrm{GeV}$ [cf. Figure 6(d)] and might also become larger if other cuts or event selection procedures are applied.

Figure 9 shows the energy distribution of $\mathrm{e}^{+}$and $\mathrm{d}$ for the process $\gamma \gamma \rightarrow \nu_{\mathrm{e}} \mathrm{e}^{+} \mathrm{d} \overline{\mathrm{u}}$. The characteristics of the Born cross section, especially the influence of the effective polarization of the photons after Compton backscattering, were explained in detail in Section 6.3 of Ref. [3]. The relative corrections shown in the lower plots amount to a few per cent. For very low and very high energies, where the Born cross section is very small, the relative corrections in DPA are not reliable anymore. In this region the assumption that doubly-resonant diagrams dominate is not fulfilled. The angular distributions for $\mathrm{e}^{+}$and $\mathrm{d}$ are shown in Figure 10. The shape of the Born cross section and the influence of the photon spectrum were also explained in Section 6.3 of Ref. [3]. Similar to the angular distributions of the $\nu_{\mathrm{e}} \mathrm{e}^{+}$and du systems, the corrections are maximal in a region where the fermions are emitted perpendicular to the beam. However, after including the photon spectrum, the corrections almost cancel as can be anticipated from 

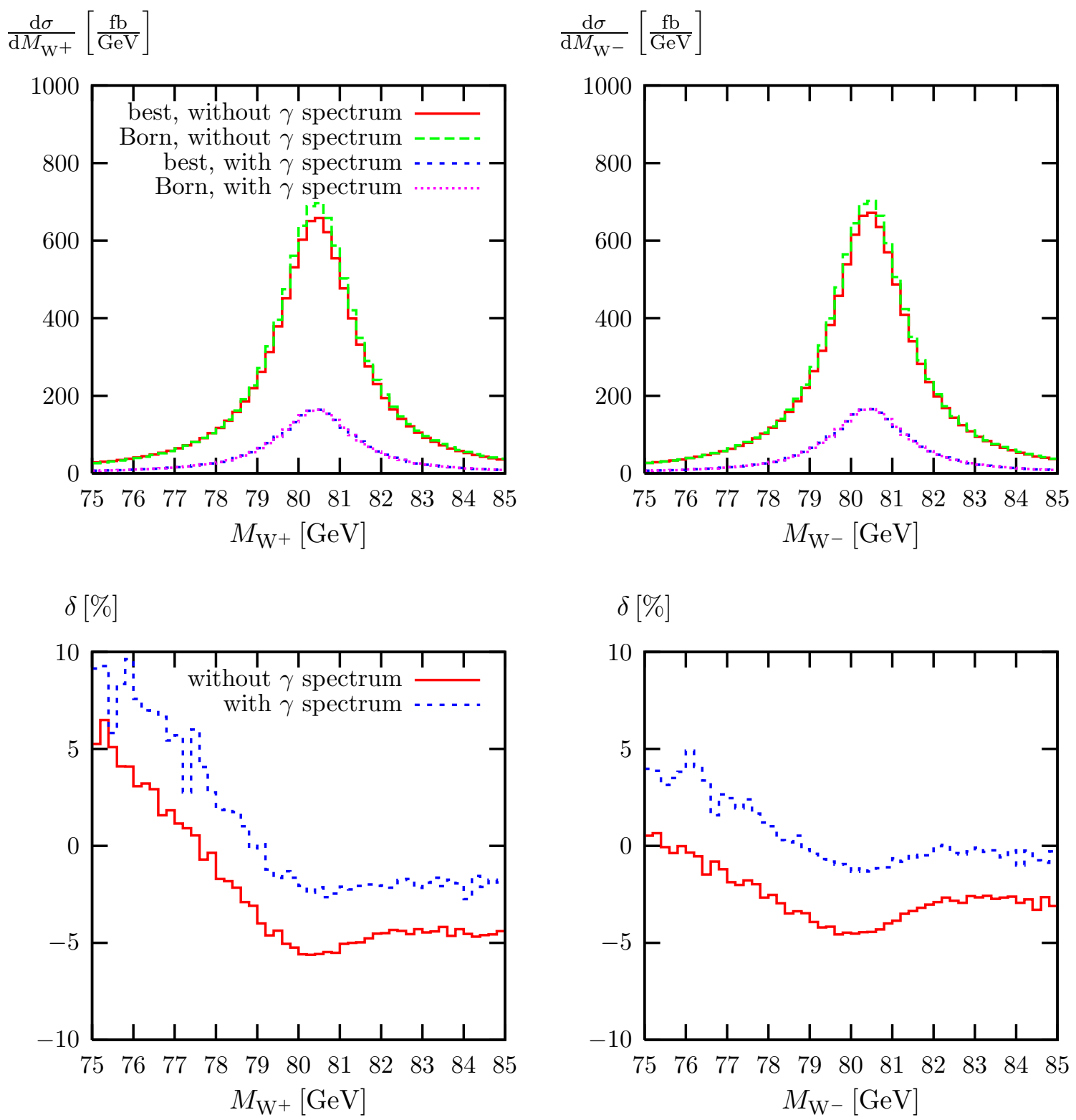

Figure 7: Invariant-mass distributions of the $\mathrm{W}^{+}$and $\mathrm{W}^{-}$bosons reconstructed from the $\nu_{\mathrm{e}} \mathrm{e}^{+}$and du pairs in the process $\gamma \gamma \rightarrow \nu_{\mathrm{e}} \mathrm{e}^{+} \mathrm{d} \overline{\mathrm{u}}$ at $\sqrt{s}=500 \mathrm{GeV}$. 

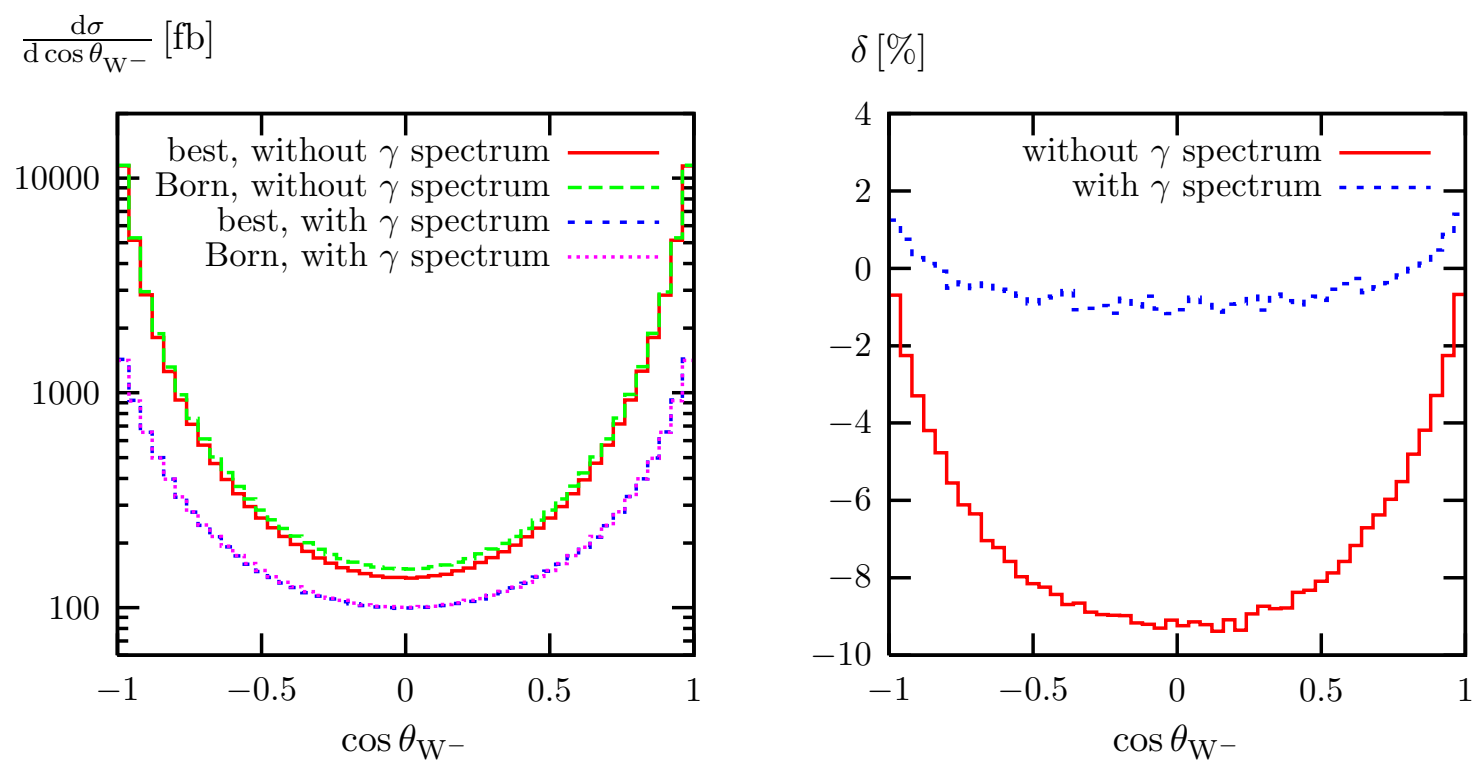

Figure 8: Angular distribution of the $\mathrm{W}^{-}$boson reconstructed from the du pair in the process $\gamma \gamma \rightarrow \nu_{\mathrm{e}} \mathrm{e}^{+} \mathrm{d} \bar{u}$ at $\sqrt{s}=500 \mathrm{GeV}$.

Figure $6(\mathrm{~d})$ which shows that the corrections to the integrated cross section are almost zero at $\sqrt{s_{\mathrm{ee}}} \sim 500 \mathrm{GeV}$.

Finally, the energy distribution of the photon in the process $\gamma \gamma \rightarrow \nu_{\mathrm{e}} \mathrm{e}^{+} \mathrm{d} \overline{\mathrm{u}}+\gamma$ is shown in Figure 11. The distribution is dominated by the soft-photon pole at $k^{0} \rightarrow 0$ and decreases rapidly at higher energies. Comparing the distributions with and without convolution over the photon spectrum, the convolution shifts the curve to lower energies, because the initial-state photons already have less energy.

\subsection{Non-collinear-safe observables}

As explained in Section 4.2, the treatment of collinear singularities in non-collinearsafe observables deserves some care. Applying the generalizations of the subtraction and the slicing methods described above, we now turn to observables without photon recombination. Apart from that, the same phase-space cuts are applied as before. In Figure 12 we show the distributions of the $\nu_{\mathrm{e}} \mathrm{e}^{+}, \nu_{\mu} \mu^{+}$, and d $\overline{\mathrm{u}}$ pairs in the processes $\gamma \gamma \rightarrow \nu_{\mathrm{e}} \mathrm{e}^{+} \mathrm{d} \overline{\mathrm{u}}$, $\nu_{\mu} \mu^{+} \mathrm{d} \overline{\mathrm{u}}$. With photon recombination the leptonic invariant masses of the two processes receive the same radiative corrections since the recombination guarantees the necessary inclusiveness so that all mass singularities cancel. If the recombination is not applied, the distributions change drastically. Note, however, that the recombination is mainly a rearrangement of events, and omitting the recombination affects the integrated cross section by less than $0.5 \%$. With decreasing invariant masses the relative corrections rise, while they are smaller at large invariant masses. The reason is that without recombination final-state radiation (which is enhanced by mass logarithms) reduces the invariant mass of the reconstructed $\mathrm{W}$ boson, thereby shifting events from the dominating resonant region to lower invariant mass values. The recombination brings most of these events back to the resonance region, because it prevents momentum loss from final-state radiation. The 

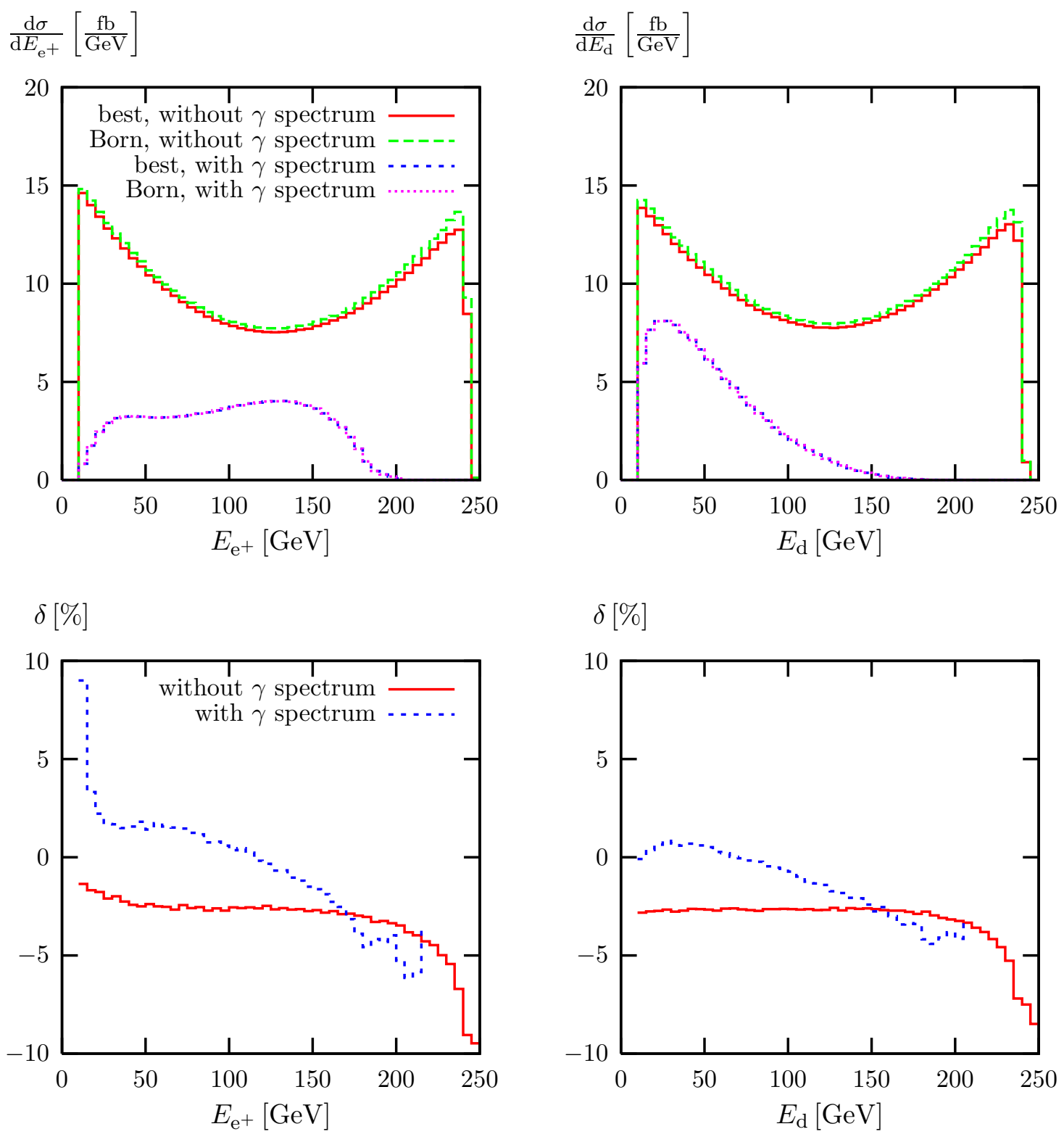

Figure 9: Energy distribution of $\mathrm{e}^{+}$and $\mathrm{d}$ in the process $\gamma \gamma \rightarrow \nu_{\mathrm{e}} \mathrm{e}^{+} \mathrm{d} \overline{\mathrm{u}}$ at $\sqrt{s}=500 \mathrm{GeV}$. 

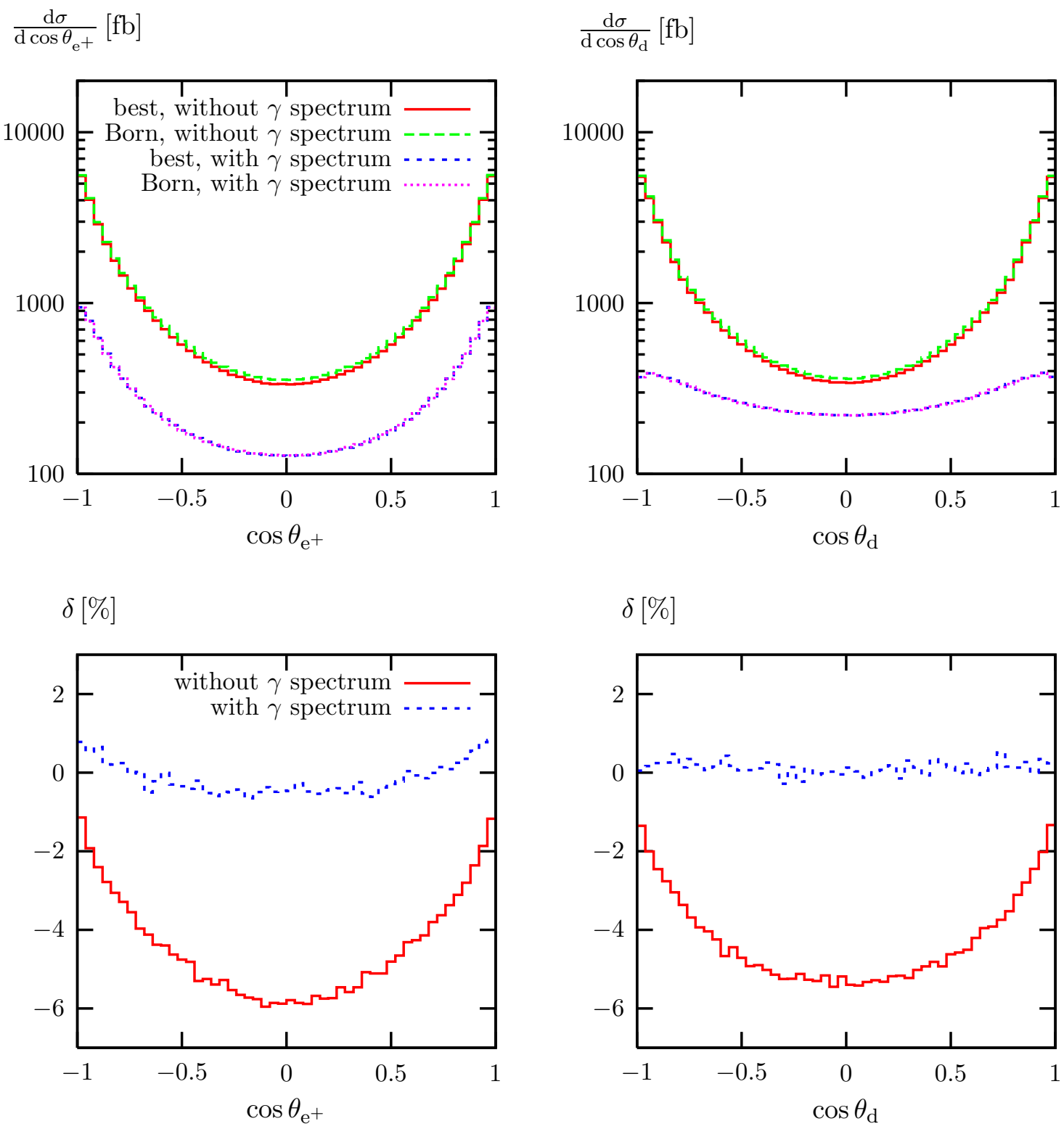

Figure 10: Distributions in the production angles of $\mathrm{e}^{+}$and $\mathrm{d}$ in the process $\gamma \gamma \rightarrow \nu_{\mathrm{e}} \mathrm{e}^{+} \mathrm{d} \overline{\mathrm{u}}$ at $\sqrt{s}=500 \mathrm{GeV}$. 

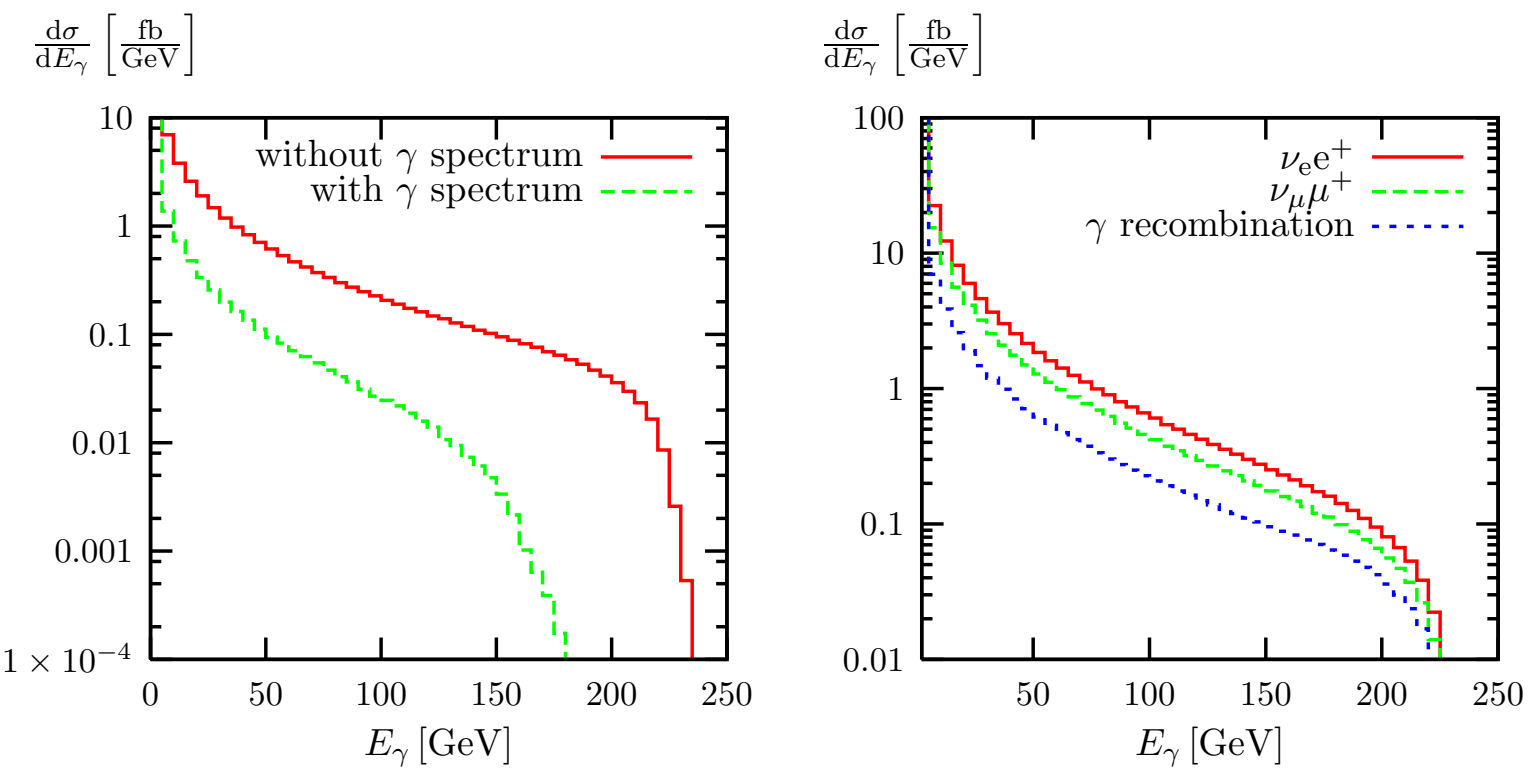

Figure 11: Energy distribution of the final-state photon in the processes $\gamma \gamma \rightarrow \nu_{\mathrm{e}} \mathrm{e}^{+} \mathrm{d} \overline{\mathrm{u}}+\gamma$ and $\gamma \gamma \rightarrow \nu_{\mu} \mu^{+}$d $\bar{u}+\gamma$ at $\sqrt{s}=500 \mathrm{GeV}$. The l.h.s. compares the distributions with and without convolution over the photon spectrum (with photon recombination); the r.h.s. compares the cases with and without photon recombination (without convolution over the photon spectrum).
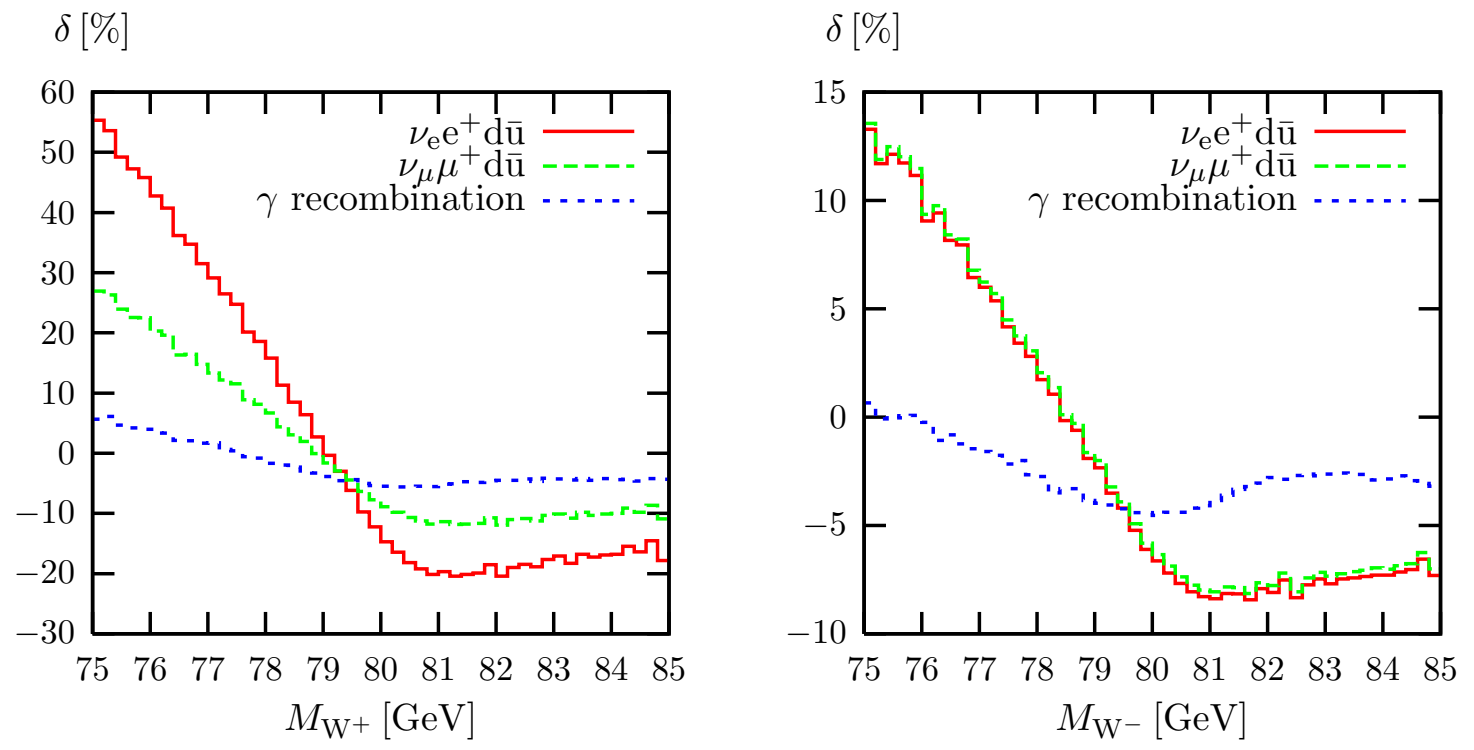

Figure 12: Invariant-mass distributions of the $\mathrm{W}^{+}$and $\mathrm{W}^{-}$boson reconstructed from the $\nu_{\mathrm{e}} \mathrm{e}^{+}\left(\nu_{\mu} \mu^{+}\right)$pair and dū pair in the process $\gamma \gamma \rightarrow \nu_{\mathrm{e}} \mathrm{e}^{+} \mathrm{d} \overline{\mathrm{u}}\left(\gamma \gamma \rightarrow \nu_{\mu} \mu^{+} \mathrm{d} \overline{\mathrm{u}}\right)$ at $\sqrt{s_{\gamma \gamma}}=$ $500 \mathrm{GeV}$, with and without photon recombination. 


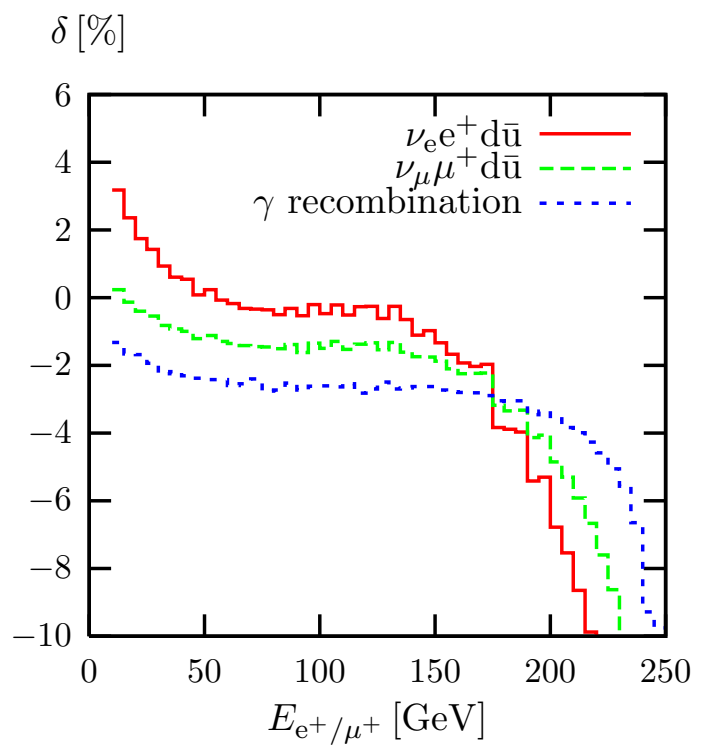

Figure 13: Energy distributions of $\mathrm{e}^{+}$and $\mu^{+}$in the processes $\gamma \gamma \rightarrow \nu_{\mathrm{e}} \mathrm{e}^{+} \mathrm{d} \overline{\mathrm{u}}$ and $\gamma \gamma \rightarrow$ $\nu_{\mu} \mu^{+} \mathrm{d} \overline{\mathrm{u}}$ at $\sqrt{s_{\gamma \gamma}}=500 \mathrm{GeV}$, with and without photon recombination.

1.h.s. of Figure 12 also shows a hierarchy in the mass effects of the outgoing leptons as the slope for the $\nu_{\mathrm{e}} \mathrm{e}^{+}$pair is much steeper than the slope for the $\nu_{\mu} \mu^{+}$pair due to the smaller mass of $\mathrm{e}^{+}$. The plot on the r.h.s. shows that the corrections for the d $\bar{u}$ pair are not as large as for the $\nu_{\mu} \mu^{+}$pair on the l.h.s., because the remaining mass terms behave like $Q_{f}^{2} \ln m_{f}$, where $Q_{f}$ denotes the charge of the fermion $f$. We also note that the corrections are smallest in the case with photon recombination because of the cancellation of all mass singularities.

The photon recombination also affects the energy distributions of the fermions. Figure 13 shows this distribution for $\mathrm{e}^{+}$and $\mu^{+}$in the processes $\gamma \gamma \rightarrow \nu_{\mathrm{e}} \mathrm{e}^{+} \mathrm{d} \overline{\mathrm{u}}$ and $\gamma \gamma \rightarrow$ $\nu_{\mu} \mu^{+} \mathrm{d} \overline{\mathrm{u}}$ with and without recombination. In the former case the curves coincide, as explained above. The recombination attributes the photon to a fermion and, thus, shifts events to higher energies of the fermion. The mass-singular effect, which appears without recombination, is again larger for $\mathrm{e}^{+}$than for $\mu^{+}$.

The effect of the photon recombination on the photon-energy spectrum is shown in Figure 11. Without recombination the distribution is shifted to higher photon energies because the recombination transfers events to the bin with zero photon energy. The difference is again bigger for the process $\gamma \gamma \rightarrow \nu_{\mathrm{e}} \mathrm{e}^{+} \mathrm{d} \overline{\mathrm{u}}$ than for $\gamma \gamma \rightarrow \nu_{\mu} \mu^{+} \mathrm{d} \overline{\mathrm{u}}$, since the mass-singular logarithms of $\mathrm{e}^{+}$are larger.

\section{Summary}

In this paper we have described a calculation of the $\mathcal{O}(\alpha)$ electroweak radiative corrections to $\gamma \gamma \rightarrow \mathrm{WW} \rightarrow 4 f$ in the electroweak Standard Model in the double-pole approximation (DPA). Technically, we follow the strategy of the RACOONWW Monte Carlo event generator for the corresponding $\mathrm{e}^{+} \mathrm{e}^{-}$reaction. This means, virtual corrections are 
treated in DPA and are decomposed into factorizable and non-factorizable contributions, while real-photonic corrections are based on complete lowest-order matrix elements for $\gamma \gamma \rightarrow 4 f \gamma$. The combination of virtual and real corrections is done in two different ways: by using the dipole subtraction method or by applying phase-space slicing.

A detailed survey of numerical results for the $\mathcal{O}(\alpha)$ corrections has been given, comprising results on integrated cross sections as well as angular, energy, and W-invariantmass distributions. In the $\mathrm{W}$-pair threshold region the corrections are dominated by the Coulomb singularity and are, thus, positive and of the order of a few per cent. For increasing $\gamma \gamma$ scattering energies the corrections become more and more negative and reach about $-10 \%$ in the $\mathrm{TeV}$ range for integrated cross sections. For large scattering angles, where the Born cross section is relatively small, the impact of the corrections is usually larger. Since the convolution with realistic photon beam spectra effectively reduces the hard scattering energy, the size of the corrections is usually somewhat reduced compared to the situation with monochromatic photon beams. Typically, collinear-safe observables (i.e. where mass-singular logarithms cancel due to sufficient inclusiveness) receive corrections of a few per cent for energies of the $\mathrm{e}^{-} \mathrm{e}^{-}$system before Compton backscattering up to $1 \mathrm{TeV}$. As expected, non-collinear-safe observables receive very large corrections (tens of per cent) because of the existence of logarithmic mass singularities.

The radiative corrections are implemented in a Monte Carlo generator called COFFER $\gamma \gamma$, which optionally includes anomalous triple and quartic gauge-boson couplings in addition and performs a convolution over realistic spectra of the photon beams. The construction of this generator and lowest-order results obtained with it have already been described in a previous publication [3]. At present, CofFER $\gamma \gamma$ is the only event generator that includes both the decays of the $\mathrm{W}$ bosons and radiative corrections, thereby defining the state-of-the-art in the description of the processes $\gamma \gamma \rightarrow \mathrm{WW} \rightarrow 4 f(+\gamma)$.

\section{Appendix}

\section{A Transformation of the coefficient functions $\boldsymbol{F}_{j}$}

In this appendix we describe the transformation of the coefficient functions $F_{j}$ for the factorizable virtual corrections (3.9) that transforms all $F_{j}$ into the helicity amplitudes of the on-shell process $\gamma \gamma \rightarrow \mathrm{WW}$.

The 36 SME $\mathcal{M}_{j}^{\gamma \gamma \mathrm{W}^{+} \mathrm{W}^{-}}$of Ref. [16], which fix the coefficient functions $F_{j}$ by Eq. (3.6), are defined for 36 different helicity configurations which can be enumerated with a single index $l$,

$$
\mathcal{M}_{j}^{\gamma \gamma \mathrm{W}^{+} \mathrm{W}^{-}}\left(\lambda_{1}, \lambda_{2} ; \lambda_{+}, \lambda_{-}\right) \equiv M_{j l}, \quad l=\left(\lambda_{1}, \lambda_{2}, \lambda_{+}, \lambda_{-}\right),
$$

where $j, l=1, \ldots, 36$. The $36 \times 36$ matrix $M$ is explicitly obtained by inserting momenta

and polarization vectors into the 36 independent $\mathrm{SME} \mathcal{M}_{j}^{\gamma \gamma \mathrm{W}^{+} \mathrm{W}^{-}}$of the 83 structures defined in Eqs. (5)-(9) of Ref. [16].

If we transform the $F_{j}$ according to

$$
\hat{F}_{l}=\sum_{j=1}^{36} F_{j} M_{j l}
$$


the function $\hat{F}_{l}$ is the helicity amplitude for the on-shell process $\gamma \gamma \rightarrow \mathrm{WW}$ corresponding to the helicity configuration $l=\left(\lambda_{1}, \lambda_{2}, \lambda_{+}, \lambda_{-}\right)$. As such, it can be well approximated by the generalized Fourier series described in Section 3.2.2. It is important to notice that in Ref. [16] the scattering plane spanned by the beam axes and the produced $\mathrm{W}$ bosons was rotated into the $\left(x^{1}, x^{3}\right)$-plane, so that the $\mathrm{SME} \mathcal{M}_{j}^{\gamma \gamma \mathrm{W}^{+} \mathrm{W}^{-}}$depend only on $s$ and $\cos \theta$, or equivalently on $s$ and $\hat{t}$. Since, thus, the matrix $M$ is a function of $s$ and $\hat{t}$, also the new functions $\hat{F}_{l}$ depend only on $s$ and $\hat{t}$, but not on the azimuthal angle of the scattering plane or other on kinematical variables. According to Eq. (A.2), the SME $\mathcal{M}_{j}$ transform as

$$
\hat{\mathcal{M}}_{l}=\sum_{j=1}^{36}\left(M^{-1}\right)_{l j} \mathcal{M}_{j}
$$

where $M^{-1}$ denotes the inverse matrix of $M$. By construction, the transformation decouples the different helicity channels of $\gamma \gamma \rightarrow$ WW. When including the W decays in the SME, as done in Eq. (A.3), this decoupling is somewhat disguised for the W-boson polarizations, but still valid for the photon helicities. This means that the new SME $\hat{\mathcal{M}}_{l}$ consist of four subsets, each of which contributes only for one of the four different polarization combinations $\left(\lambda_{1}, \lambda_{2}\right)$ of the photons. In practice, we have evaluated and simplified the matrix $M$ and the new SME $\hat{\mathcal{M}}_{l}$ analytically as much as possible.

\section{B Dipole subtraction for non-collinear-safe photonic final-state radiation}

In Section 4.2.2 we have collected the relevant formulas for the generalization of the dipole subtraction method to non-collinear-safe observables. Here we describe the details of their derivation. Specifically, we focus on the situation of light charged particles in the final state only; the more general case of massive particles and of charged particles in the initial state will be worked out elsewhere [36]. Although not made explicit in the main text, we keep track of the polarizations of the outgoing particles.

\section{(i) Subtraction of singularities}

Generically the schematic form of the subtraction procedure to integrate the squared matrix element $\sum_{\lambda_{\gamma}}\left|\mathcal{M}_{1}\right|^{2}$ (summed over the photon polarizations $\lambda_{\gamma}$ ) for real photon radiation over the $(N+1)$-particle phase space $\mathrm{d} \Phi_{1}$ reads

$$
\int \mathrm{d} \Phi_{1} \sum_{\lambda_{\gamma}}\left|\mathcal{M}_{1}\right|^{2}=\int \mathrm{d} \Phi_{1}\left(\sum_{\lambda_{\gamma}}\left|\mathcal{M}_{1}\right|^{2}-\left|\mathcal{M}_{\text {sub }}\right|^{2}\right)+\int \mathrm{d} \tilde{\Phi}_{0} \otimes\left(\int[\mathrm{d} k]\left|\mathcal{M}_{\text {sub }}\right|^{2}\right),
$$

where $\mathrm{d} \tilde{\Phi}_{0}$ is a phase-space element of the corresponding non-radiative process and [d $k$ ] includes the photonic phase space that leads to the soft and collinear singularities. The sign " $\otimes$ " indicates that this factorization, in general, is not an ordinary product, but may contain also summations and convolutions. The two contributions involving the subtraction function $\left|\mathcal{M}_{\text {sub }}\right|^{2}$ have to cancel each other, however, they will be evaluated separately. The subtraction function is constructed in such a way that the difference $\left(\sum_{\lambda_{\gamma}}\left|\mathcal{M}_{1}\right|^{2}-\left|\mathcal{M}_{\text {sub }}\right|^{2}\right)$ can be safely integrated over $\mathrm{d} \Phi_{1}$ numerically and that the singular integration of $\left|\mathcal{M}_{\text {sub }}\right|^{2}$ over $[\mathrm{d} k]$ can be carried out analytically, followed by a safe numerical integration over $\mathrm{d} \tilde{\Phi}_{0}$. 
In the dipole subtraction formalism, the subtraction function is given by $[22,23]$

$$
\left|\mathcal{M}_{\mathrm{sub}}\left(\Phi_{1}\right)\right|^{2}=-\sum_{i \neq j} Q_{i} \sigma_{i} Q_{j} \sigma_{j} e^{2} g_{i j, \tau}^{(\mathrm{sub})}\left(p_{i}, p_{j}, k\right)\left|\mathcal{M}_{0}\left(\tilde{\Phi}_{0, i j} ; \tau \kappa_{i}\right)\right|^{2}
$$

where the sum runs over all emitter-spectator pairs $i j$, which are called dipoles. Recall that both $i$ and $j$ are final-state particles in our case. The relative charges are denoted $Q_{f}$ $(f=i, j)$, and the sign factors $\sigma_{f}= \pm 1$ correspond to the charge flow $\left(\sigma_{f}=+1\right.$ for antifermions, $\sigma_{f}=-1$ for fermions). The summation over $\tau= \pm 1$ accounts for a possible flip in the helicity $\kappa_{i}$ of the emitter $i$. The singular behaviour of the subtraction function is contained in the radiator functions $g_{i j, \tau}^{(\mathrm{sub})}\left(p_{i}, p_{j}, k\right)$, which depend on the emitter, spectator, and photon momenta $p_{i}, p_{j}$, and $k$, respectively. In the limit of small fermion masses the functions $g_{i j, \tau}^{(\mathrm{sub})}$ are related to the function $g_{i j}^{(\mathrm{sub})}$ of Eq. (4.7) for the unpolarized case by $g_{i j,+}^{(\mathrm{sub})}=g_{i j}^{(\mathrm{sub})}, g_{i j,-}^{(\mathrm{sub})}=0$. The squared lowest-order matrix element $\left|\mathcal{M}_{0}\right|^{2}$ of the corresponding non-radiative process enters the subtraction function with modified emitter and spectator momenta $\tilde{p}_{i}, \tilde{p}_{j}$, as defined in Eq. (4.9). The momenta are related by $p_{i}+p_{j}+k=\tilde{p}_{i}+\tilde{p}_{j}$, where all the other particle momenta $p_{k}$ and $\tilde{p}_{k}$, entering $\left|\mathcal{M}_{1}\right|^{2}$ and $\left|\mathcal{M}_{0}\right|^{2}$, respectively, are the same, $\tilde{p}_{k}=p_{k}$. The modified momenta are constructed in such a way that $\tilde{p}_{i} \rightarrow p_{i}+k$ in the collinear limit $\left(p_{i} k \rightarrow 0\right)$. Since we deal with light external fermions only, we set all masses $m_{f}$ of external fermions to zero whenever possible. This means that $m_{f}=0$ can be consistently used in the integral $\int \mathrm{d} \Phi_{1}\left(\sum_{\lambda_{\gamma}}\left|\mathcal{M}_{1}\right|^{2}-\left|\mathcal{M}_{\text {sub }}\right|^{2}\right)$, but that the readded contribution $\int[\mathrm{d} k]\left|\mathcal{M}_{\text {sub }}\right|^{2}$ in general contains mass-singular terms of the form $\alpha \ln m_{f}$.

In collinear-safe observables, and only those are considered for light fermions in Refs. [22,23], a collinear fermion-photon system is treated as one quasi-particle, i.e., in the limit where a charged fermion $i$ and $\gamma$ become collinear only the sum $p_{i}+k$ enters the procedures of implementing phase-space selection cuts or of sorting an event into a histogram bin of a differential distribution. Technically this level of inclusiveness is reached by photon recombination, a procedure that assigns the photon to the nearest charged particle if it is close enough to it. Of course, different variants for such an algorithm are possible, similar to jet algorithms in QCD. The recombination guarantees that for each photon radiation cone around a charged particle $i$ the energy fraction of Eq. (4.14), $z_{i}=p_{i}^{0} /\left(p_{i}^{0}+k^{0}\right)$, is fully integrated over. According to the KLN theorem [33], no mass singularity connected with final-state radiation remains. Collinear safety facilitates the actual application of the subtraction procedure as indicated in Eq. (A.1). In this case the events resulting from the contributions of $\left|\mathcal{M}_{\text {sub }}\right|^{2}$ can be consistently regarded as $N$-particle final states of the non-radiative process with particle momenta as going into $\left|\mathcal{M}_{0}\left(\tilde{\Phi}_{0, i j}\right)\right|^{2}$, i.e. the emitter and spectator momenta are given by $\tilde{p}_{i}, \tilde{p}_{j}$, respectively. Owing to $\tilde{p}_{i} \rightarrow p_{i}+k$ in the collinear limits, the difference $\left(\sum_{\lambda_{\gamma}}\left|\mathcal{M}_{1}\right|^{2}-\left|\mathcal{M}_{\text {sub }}\right|^{2}\right)$ can be integrated over all collinear regions, because all events that differ only in the value of $z_{i}$ enter cuts or histograms in the same way. The implicit full integration over all $z_{i}$ in the collinear cones, on the other hand, implies that in the analytical integration of $\left|\mathcal{M}_{\text {sub }}\right|^{2}$ over $[\mathrm{d} k]$ the $z_{i}$ integrations can be carried out over the whole $z_{i}$ range.

In non-collinear-safe observables, not all photons within arbitrarily narrow collinear cones around outgoing charged particles are treated inclusively. For a fixed cone axis 
the integration over the corresponding variable $z_{i}$ is constrained by a phase-space cut or by the boundary of a histogram bin. Consequently, mass-singular contributions of the form $\alpha \ln m_{i}$ remain in the integral. Technically this means that the information on the variables $z_{i}$ has to be exploited in the subtraction procedure of Eq. (A.1). The variable that takes over the role of $z_{i}$ in the individual dipole contributions in $\left|\mathcal{M}_{\text {sub }}\right|^{2}$ is $z_{i j}$, as defined in Eq. (4.8), because in the collinear limit it behaves as $z_{i j} \rightarrow z_{i}$. Thus, the integral $\int \mathrm{d} \Phi_{1}\left(\sum_{\lambda_{\gamma}}\left|\mathcal{M}_{1}\right|^{2}-\left|\mathcal{M}_{\text {sub }}\right|^{2}\right)$ can be performed over the whole phase space if the events associated with $\left|\mathcal{M}_{\text {sub }}\right|^{2}$ are treated as $(N+1)$-particle events with momenta $p_{i}=z_{i j} \tilde{p}_{i}$, $p_{j}=\tilde{p}_{j}$, and $k=\left(1-z_{i j}\right) \tilde{p}_{i}$. This modification, in turn, requires a generalization in the evaluation of the second subtraction term on the r.h.s. of Eq. (A.1), because now the integral over $z_{i j}$ implicitly contained in $[\mathrm{d} k]$ depends on the cuts that define the observable.

\section{(ii) Integration of singular contributions}

For a final-state emitter $i$ and a final-state spectator $j$ with masses $m_{i}$ and $m_{j}$ the integral of $g_{i j, \tau}^{(\mathrm{sub})}\left(p_{i}, p_{j}, k\right)$ over $[\mathrm{d} k]$ is proportional to

$$
G_{i j, \tau}^{(\mathrm{sub})}\left(P_{i j}^{2}\right)=\frac{\bar{P}_{i j}^{4}}{2 \sqrt{\lambda_{i j}}} \int_{y_{1}}^{y_{2}} \mathrm{~d} y_{i j}\left(1-y_{i j}\right) \int_{z_{1}\left(y_{i j}\right)}^{z_{2}\left(y_{i j}\right)} \mathrm{d} z_{i j} g_{i j, \tau}^{(\mathrm{sub})}\left(p_{i}, p_{j}, k\right),
$$

where $P_{i j}^{2}=\left(\tilde{p}_{i}+\tilde{p}_{j}\right)^{2}$ and the definitions of Section 4.1 of Ref. [22] are used. There the

results for $G_{i j, \tau}^{(\mathrm{sub})}\left(P_{i j}^{2}\right)$ with generic or light masses are given in Eqs. (4.10) and (3.7), respectively. In order to leave the integration over $z_{i j}$ open, the order of the two integrations has to be interchanged, and the integral solely taken over $y_{i j}$ is needed,

$$
\overline{\mathcal{G}}_{i j, \tau}^{(\mathrm{sub})}\left(P_{i j}^{2}, z_{i j}\right)=\frac{\bar{P}_{i j}^{4}}{2 \sqrt{\lambda_{i j}}} \int_{y_{1}\left(z_{i j}\right)}^{y_{2}\left(z_{i j}\right)} \mathrm{d} y_{i j}\left(1-y_{i j}\right) g_{i j, \tau}^{(\mathrm{sub})}\left(p_{i}, p_{j}, k\right) .
$$

Note that the function $\overline{\mathcal{G}}_{i j, \tau}^{(\mathrm{sub})}\left(P_{i j}^{2}, z\right)$ is not needed for finite photon mass $\lambda$, because the soft singularity appearing at $z \rightarrow 1$ can be split off by employing a $[\ldots]_{+}$prescription in the variable $z$,

$$
\overline{\mathcal{G}}_{i j, \tau}^{(\mathrm{sub})}\left(P_{i j}^{2}, z\right)=G_{i j, \tau}^{(\mathrm{sub})}\left(P_{i j}^{2}\right) \delta(1-z)+\left[\overline{\mathcal{G}}_{i j, \tau}^{(\mathrm{sub})}\left(P_{i j}^{2}, z\right)\right]_{+} .
$$

This procedure shifts the soft singularity into the quantity $G_{i j, \tau}^{(\mathrm{sub})}\left(P_{i j}^{2}\right)$, which is already known from Ref. [22]. Moreover, the generalization to non-collinear-safe integrals simply reduces to the extra term $\left[\overline{\mathcal{G}}_{i j, \tau}^{(\mathrm{sub})}\left(P_{i j}^{2}, z\right)\right]_{+}$, which cancels out for collinear-safe integrals where the full $z$-integration is carried out. by

In the limit $m_{i} \rightarrow 0$ and for $m_{j}=\lambda=0$ the boundary of the $y_{i j}$ integration is given

$$
y_{1}(z)=\frac{m_{i}^{2}(1-z)}{P_{i j}^{2} z}, \quad y_{2}(z)=1,
$$

and the functions relevant for the integrand $g_{i j, \tau}^{(\mathrm{sub})}$ behave as (see Section 4.1 of Ref. [22])

$$
p_{i} k=\frac{P_{i j}^{2}}{2} y_{i j}, \quad R_{i j}(y)=1-y, \quad r_{i j}(y)=1
$$


The evaluation of Eq. (A.4) becomes very simple and yields

$$
\begin{aligned}
& \overline{\mathcal{G}}_{i j,+}^{\text {(sub) }}\left(P_{i j}^{2}, z\right)=P_{f f}(z)\left[\ln \left(\frac{P_{i j}^{2} z}{m_{i}^{2}}\right)-1\right]+(1+z) \ln (1-z), \\
& \overline{\mathcal{G}}_{i j,-}^{\text {(sub) }}\left(P_{i j}^{2}, z\right)=1-z,
\end{aligned}
$$

where $P_{f f}(y)$ is the splitting function of Eq. (4.16). Equation (A.8) is correct up to terms suppressed by factors of $m_{i}$. For completeness, we repeat the form of the full integral $G_{i j, \tau}^{(\mathrm{sub})}\left(P_{i j}^{2}\right)$ in the case of light masses,

$$
G_{i j,+}^{(\mathrm{sub})}\left(P_{i j}^{2}\right)=\mathcal{L}\left(P_{i j}^{2}, m_{i}^{2}\right)-\frac{\pi^{2}}{3}+1, \quad G_{i j,-}^{(\mathrm{sub})}\left(P_{i j}^{2}\right)=\frac{1}{2},
$$

with the auxiliary function $\mathcal{L}$ of Eq. (4.13). The results for the functions $\overline{\mathcal{G}}_{i j}^{(\text {sub })}$ and $G_{i j}^{(\text {sub })}$ for the unpolarized case, as given in Eqs. (4.18) and (4.12), are obtained by summing over the variable $\tau= \pm 1$ which accounts for the spin-flip,

$$
\overline{\mathcal{G}}_{i j}^{(\mathrm{sub})}=\overline{\mathcal{G}}_{i j,+}^{(\mathrm{sub})}+\overline{\mathcal{G}}_{i j,-}^{(\mathrm{sub})}, \quad G_{i j}^{(\mathrm{sub})}=G_{i j,+}^{(\mathrm{sub})}+G_{i j,-}^{(\mathrm{sub})} .
$$

Finally, we give the explicit form of the $i j$ contribution $\left|\mathcal{M}_{\text {sub }, i j}\left(\Phi_{1}\right)\right|^{2}$ to the phasespace integral of the subtraction function,

$$
\begin{aligned}
& \int \mathrm{d} \Phi_{1}\left|\mathcal{M}_{\text {sub }, i j}\left(\Phi_{1}\right)\right|^{2}=-\frac{\alpha}{2 \pi} Q_{i} \sigma_{i} Q_{j} \sigma_{j} \int \mathrm{d} \tilde{\Phi}_{0, i j}\left|\mathcal{M}_{0}\left(\tilde{p}_{i}, \tilde{p}_{j} ; \tau \kappa_{i}\right)\right|^{2} \\
& \quad \times\left\{G_{i j, \tau}^{\text {(sub) }}\left(P_{i j}^{2}\right) \Theta\left(\tilde{\Phi}_{0, i j}\right)+\int_{0}^{1} \mathrm{~d} z\left[\overline{\mathcal{G}}_{i j, \tau}^{(\text {sub })}\left(P_{i j}^{2}, z\right)\right]_{+} \Theta\left(p_{i}=z \tilde{p}_{i}, k=(1-z) \tilde{p}_{i},\left\{\tilde{p}_{k \neq i}\right\}\right)\right\} .
\end{aligned}
$$

The arguments of the step functions $\Theta(\ldots)$ indicate on which momenta phase-space cuts are imposed.

\section{References}

[1] I. F. Ginzburg et al., Nucl. Instrum. Meth. 205 (1983) 47 and Nucl. Instrum. Meth. A 219 (1984) 5;

V. I. Telnov, Nucl. Instrum. Meth. A 294 (1990) 72;

R. Brinkmann et al., Nucl. Instrum. Meth. A 406 (1998) 13 [hep-ex/9707017];

I. Watanabe et al., " $\gamma \gamma$ collider as an option of JLC," KEK-REPORT-97-17;

B. Badelek et al. "TESLA Technical Design Report, Part VI, Chapter 1: Photon collider at TESLA," hep-ex/0108012;

T. Abe et al. [American Linear Collider Working Group Collaboration], in Proc. of the APS/DPF/DPB Summer Study on the Future of Particle Physics (Snowmass 2001) ed. R. Davidson and C. Quigg, SLAC-R-570, Resource book for Snowmass 2001 [hep-ex/0106055, hep-ex/0106056, hep-ex/0106057, hep-ex/0106058].

[2] A. De Roeck, hep-ph/0311138;

M. Krawczyk, hep-ph/0312341;

S. J. Brodsky, hep-ph/0404186. 
[3] A. Bredenstein, S. Dittmaier and M. Roth, Eur. Phys. J. C 36 (2004) 341 [hep$\mathrm{ph} / 0405169]$.

[4] A. F. Zarnecki, Acta Phys. Polon. B 34 (2003) 2741 [hep-ex/0207021].

[5] W. Kilian, "WHIZARD 1.0: A generic Monte-Carlo integration and event generation package for multi-particle processes. Manual," LC-TOOL-2001-039.

[6] T. Stelzer and W. F. Long, Comput. Phys. Commun. 81 (1994) 357 [hep-ph/9401258]. H. Murayama, I. Watanabe and K. Hagiwara, "HELAS: HELicity amplitude subroutines for Feynman diagram evaluations," KEK-91-11.

[7] A. Denner, S. Dittmaier, M. Roth and L. H. Wieders, Phys. Lett. B 612 (2005) 223 [hep-ph/0502063].

[8] A. Denner, S. Dittmaier, M. Roth and L. H. Wieders, hep-ph/0505042.

[9] A. Denner, S. Dittmaier, M. Roth and D. Wackeroth, Nucl. Phys. B 560 (1999) 33 [hep-ph/9904472]; Phys. Lett. B 475 (2000) 127 [hep-ph/9912261]; Eur. Phys. J. direct C 2 (2000) 4 [hep-ph/9912447]; hep-ph/0101257 and Comput. Phys. Commun. 153 (2003) 462 [hep-ph/0209330].

[10] A. Denner, S. Dittmaier, M. Roth and D. Wackeroth, Nucl. Phys. B 587 (2000) 67 [hep-ph/0006307].

[11] A. Aeppli, G. J. van Oldenborgh and D. Wyler, Nucl. Phys. B 428 (1994) 126 [hep$\mathrm{ph} / 9312212]$.

[12] S. Jadach et al., Phys. Rev. D 61 (2000) 113010 [hep-ph/9907436]; Phys. Rev. D 65 (2002) 093010 [hep-ph/0007012]; Comput. Phys. Commun. 140 (2001) 432 [hepph/0103163] and Comput. Phys. Commun. 140 (2001) 475 [hep-ph/0104049].

[13] W. Beenakker, F. A. Berends and A. P. Chapovsky, Nucl. Phys. B 548 (1999) 3 [hep-ph/9811481].

[14] Y. Kurihara, M. Kuroda and D. Schildknecht, Phys. Lett. B 509 (2001) 87 [hep$\mathrm{ph} / 0104201]$.

[15] A. Denner, S. Dittmaier and R. Schuster, Phys. Rev. D 51 (1995) 4738 [hep$\mathrm{ph} / 9411268]$.

[16] A. Denner, S. Dittmaier and R. Schuster, Nucl. Phys. B 452 (1995) 80 [hep$\mathrm{ph} / 9503442]$.

[17] G. Jikia, Nucl. Phys. B 494 (1997) 19 [hep-ph/9612380].

[18] I. B. Marfin, V. A. Mossolov and T. V. Shishkina, hep-ph/0305153 and hep$\mathrm{ph} / 0401068$. 
[19] D. Y. Bardin, S. Riemann and T. Riemann, Z. Phys. C 32 (1986) 121;

F. Jegerlehner, Z. Phys. C 32 (1986) 425 [Erratum-ibid. C 38 (1988) 519];

A. Denner and T. Sack, Z. Phys. C 46 (1990) 653.

[20] K. Melnikov and O. I. Yakovlev, Nucl. Phys. B 471 (1996) 90 [hep-ph/9501358];

W. Beenakker, A. P. Chapovsky and F. A. Berends, Phys. Lett. B 411 (1997) 203 [hep-ph/9706339] and Nucl. Phys. B 508 (1997) 17 [hep-ph/9707326].

[21] A. Denner, S. Dittmaier and M. Roth, Nucl. Phys. B 519 (1998) 39 [hep-ph/9710521].

[22] S. Dittmaier, Nucl. Phys. B 565 (2000) 69 [hep-ph/9904440].

[23] M. Roth, PhD thesis, ETH Zürich No. 13363 (1999), hep-ph/0008033.

[24] J. Küblbeck, M. Böhm and A. Denner, Comput. Phys. Commun. 60 (1990) 165;

H. Eck and J. Küblbeck, Guide to FeynArts 1.0, University of Würzburg, 1992.

[25] A. Denner, S. Dittmaier and G. Weiglein, Nucl. Phys. B 440 (1995) 95 [hep$\mathrm{ph} / 9410338]$.

[26] S. Dittmaier, Phys. Rev. D 59 (1999) 016007 [hep-ph/9805445].

[27] G. Passarino and M. J. G. Veltman, Nucl. Phys. B 160 (1979) 151.

[28] A. Denner, Fortsch. Phys. 41 (1993) 307.

[29] A. Djouadi, M. Spira, J. J. van der Bij and P. M. Zerwas, Phys. Lett. B 257 (1991) 187.

A. Djouadi, M. Spira and P. M. Zerwas, Phys. Lett. B 311 (1993) 255 [hep$\mathrm{ph} / 9305335]$.

K. Melnikov and O. I. Yakovlev, Phys. Lett. B 312 (1993) 179 [hep-ph/9302281].

M. Inoue, R. Najima, T. Oka and J. Saito, Mod. Phys. Lett. A 9 (1994) 1189.

J. Fleischer, O. V. Tarasov and V. O. Tarasov, Phys. Lett. B 584 (2004) 294 [hep$\mathrm{ph} / 0401090] ;$

U. Aglietti, R. Bonciani, G. Degrassi and A. Vicini, Phys. Lett. B 595 (2004) 432 [hep-ph/0404071].

G. Degrassi and F. Maltoni, hep-ph/0504137.

[30] A. Sirlin, Phys. Rev. D 22 (1980) 971;

W. J. Marciano and A. Sirlin, Phys. Rev. D 22 (1980) 2695 [Erratum-ibid. D 31 (1985) 213] and Nucl. Phys. B 189 (1981) 442.

[31] D. Y. Bardin, W. Beenakker and A. Denner, Phys. Lett. B 317 (1993) 213.

[32] S. Catani and M. H. Seymour, Phys. Lett. B 378 (1996) 287 [hep-ph/9602277] and Nucl. Phys. B 485 (1997) 291 [Erratum-ibid. B 510 (1997) 291] [hep-ph/9605323].

[33] T. Kinoshita, J. Math. Phys. 3 (1962) 650.

T. D. Lee and M. Nauenberg, Phys. Rev. 133 (1964) B1549. 
[34] K. Hagiwara et al. [Particle Data Group Collaboration], Phys. Rev. D 66 (2002) 010001.

[35] A. Djouadi, J. Kalinowski and M. Spira, Comput. Phys. Commun. 108 (1998) 56 [hep-ph/9704448].

[36] S. Dittmaier, in preparation. 